\title{
聚集态分子排列对光电性能的影响
}

\author{
王金凤 $a$ 李振 $*, a, b, c$ \\ ( ${ }^{a}$ 天津大学 分子聚集态科学研究院 天津 300072) \\ ( ${ }^{b}$ 武汉大学化学与分子科学学院 武汉 430072) \\ (c 天津大学-新加坡国立大学福州联合学院 福州 350207)
}

\begin{abstract}
摘要 有机小分子材料是由许多分子构成的聚集体, 其性能既与单个分子的空间构型和电子特性有关, 更与聚集体中 分子的排列方式和分子间相互作用密切相关. 在有机小分子材料中, 聚集体的性质并不是组成它的单分子行为的简单 线性叠加, 而是经常呈现出聚集态的整体差异性响应特点, 即: 不同的聚集态分子排列引起截然不同的性能. 因此, 科学研究已逐渐由单分子研究迈向分子聚集态科学. 随着人们对分子聚集态科学的关注, 特别是对有机分子固态下的 排列和堆积方式、分子间相互作用等方面的深入研究发现，通过不同策略对分子聚集态行为的有效调控，可以实现完 全不同于单个分子特性的聚集态发光现象，包括发光强度、颜色、形式以及激发过程的差异. 也可以通过分子聚集态 的形貌和维度的调节, 实现不同光电器件性能的调节和优化. 本综述将分别介绍聚集态分子的排列方式对力致变色、 室温磷光、力致发光和有机场效应晶体管等方面的重要影响, 进一步阐述聚集态分子行为的重要性, 同时, 为有效调控 聚集态分子的排列方式, 有针对性地设计和开发优异性能的光电材料提供基础.
\end{abstract}

关键词 分子排列; 力致变色; 室温磷光; 力致发光; 有机场效应晶体管

\section{Significant Influence of Molecular Packing in Aggregates on Optoelectronic Properties}

\author{
Jinfeng Wang ${ }^{a} \quad$ Zhen $\mathrm{Li}^{*, a, b, c}$ \\ ( ${ }^{a}$ Institute of Molecular Aggregation Science, Tianjin University, Tianjin 300072, China) \\ ( ${ }^{b}$ College of Chemistry and Molecular Sciences, Wuhan University, Wuhan 430072, China) \\ ( ${ }^{c}$ Joint School of National University of Singapore and Tianjin University, Fuzhou 350207, China)
}

\begin{abstract}
Organic materials based on small molecules are aggregates that composed of many molecules. Their properties are not only determined by the spatial configuration and electronic properties of individual molecule, but also closely related to the molecular packing modes and the intermolecular interactions in the aggregates. In the realm of organic molecules, the macroscopic performance of molecular aggregates is not just the simple superposition of single molecules, but often display overall differential response of aggregates, namely, distinct properties can be created by molecular uniting with particular packing modes. Therefore, molecular aggregation science has gradually appeared in scientific research and drawn much attention in recent years. With the continuous and serious exploration of molecular aggregation science, especially the in-depth study of the molecular packing modes and intermolecular interactions of organic molecules in the solid state, it is found that the luminescence based on aggregation state is completely different from the characteristics of individual molecule, including the emission intensity and color, emission type, and even the excitation process, which could be realized by regulating molecular aggregation behavior. In addition, the morphology and dimensions of the aggregates can also be adjusted to achieve excellent performances of optoelectronic devices. This review will introduce the important influence of the arrangement of aggregated molecules on mechanochromism, room temperature phosphorescence, mechanoluminescence and organic field effect transistors, and further elaborate the importance of the molecular packing, providing some guidance for regulating molecular packing mode and targeted design and development of high-performance optoelectronic materials.
\end{abstract}

Keywords molecular packing; mechanochromism; room temperature phosphorescence; mechanoluminescence; organic field effect transistor

\section{1 引言}

现代科学技术的进步与新型材料的发展和应用息 息相关, 具有优异性能和特定功能的新材料已成为智能
信息、航空航天、能源和生物等高科技领域发展的基础 材料. 其中, 有机光电功能材料作为核心部分, 因其结 构和性能可调、柔韧轻巧、加工简便、成本低廉等优点

\footnotetext{
*E-mail: lizhen@tju.edu.cn, lizhen@whu.edu.cn

Received January 28, 2021; published March 16, 2021.

Project supported by Starting Foundation of Tianjin University.

项目受天津大学启动基金资助.
} 
成为人们广泛研究的热点 ${ }^{[1-4]}$.

对于纯有机小分子发光材料而言, 通常在稀溶液中 进行发光过程的常规研究, 当扩展到聚集态时, 这些分 子的发光行为通常会发生很大变化. 实际上, 不仅单分 子特性影响材料的光电性能, 聚集态分子的排列也是影 响材料光电性能的重要因素. 更为重要的是, 大多数的 光电功能材料都是以薄膜或纳米颗粒等聚集态的形式 应用于各种光电器件和生物体系中 ${ }^{[5-6]}$, 例如, 在有机发 光二极管(OLED)和场效应晶体管中, 光电功能材料大 都是以固态薄膜的形式存在 ${ }^{[7-9]}$. 在生物成像中, 光电功 能材料通常以纳米颗粒的形式存在于生物体内 ${ }^{[10-11]}$. 而 分子堆积作为聚集态规整结构的精细展现, 是深度理解 宏观光电性能与微观分子聚集状态关联性的核心内容. 聚集态分子的不同堆积模式能够导致分子间相互作用 的差异, 并对有机光电功能材料的性能产生重要影 响 ${ }^{[12-17]}$. 本综述将从聚集态分子排列对力致变色 (MC, mechanochromic luminescent)、力致发光 (ML, mechanoluminescence)、室温磷光 (RTP, room temperature phosphorescence)和有机场效应晶体管(OFET, organic field-effect transistor)等方面的影响进行总结.

\section{2 分子排列对压(力)致变色的影响}

有机固体分子的发光性质与其分子排列方式、分子 构象以及分子间相互作用密切相关, 力刺激作用导致聚 集态分子堆积方式、构象和分子间相互作用发生改变, 并影响有机分子-电子跃迁的能级水平, 引起发光颜色 在受力前后出现差异. 聚集态分子堆积模式的改变包含 多种类型, 如: 分子堆积的滑动或改变、晶形到无定形 态、或者晶形与晶形、无定形到晶形之间的转换等. 具 有 $\operatorname{AIE}$ (聚集诱导发光)效应的扭曲单元, 如: 四苯乙烯 (TPE)、三苯乙烯单元等, 这种具有扭曲片段的分子在晶 态中的 $\pi \cdots \pi$ 相互作用较弱, 分子排列相对疏松, 在外力 的作用下, 分子排列很容易发生改变, 产生力致变色现 象 ${ }^{[18-25]}$.

2011 年, Chi 等 ${ }^{[26]}$ 报道了己基吩噻嗪取代的 9,10-二 乙烯基葸 AIE 化合物 AnPh 的力致变色性能(图 1a), 研 究发现, 研磨前后分子的发射由绿光 $(\lambda=562 \mathrm{~nm})$ 红移 到红橙光 $(\lambda=606 \mathrm{~nm})$, 并且研磨后样品可以通过热退 火和溶剂熏蒸等方式恢复. 通过晶体结构分析发现, $\mathrm{AnPh}$ 分子以 “头碰头” 的方式堆积. 由于己基吩噻嗪 的扭曲构象和空间位阻效应, 在晶态中, 相邻分子间以 较弱的 $\mathrm{C}-\mathrm{H} \cdots \mathrm{S} 、 \mathrm{~S} \cdots \pi$ 和 $\mathrm{C}-\mathrm{H} \cdots \pi$ 作用排列成层, 分子间几 乎无 $\pi \cdots \pi$ 相互作用. 而层与层间则以较弱的 $\pi \cdots \pi$ 相互作 用形成松散的分子堆积, 同时, 该化合物在单晶结构中 呈现出更加扭曲的构象, 晶体中分子的共轭程度低, 发 光波长更短. 当外力刺激时, 这种分子间扭曲应力在外 部压力刺激时被释放，晶体层与层间发生滑移，破坏了 晶体结构的长程有序排列, 导致聚集态分子排列发生改
变, 并伴随发光颜色的变化. 2015 年, Misra 等 ${ }^{[27]}$ 报道了 一种具有 AIE 性质的力致变色材料 $\mathbf{3 a}$ (图 1b), 力刺激 时, 发光颜色由蓝色变为绿色. 实验结果表明, 四苯乙 烯的引入有效减弱了分子间 $\pi \cdots \pi$ 相互作用，相邻分子形 成的二聚体间通过较弱的 $\mathrm{C}-\mathrm{H} \cdots \pi$ 相互作用形成 “头对 尾”排列，整体表现出由二聚体组成的松散二维阶梯状 排列. 这种松散的排列在力刺激作用下易于被破坏，导 致堆积方式的改变, 从而引起发光颜色的变化.

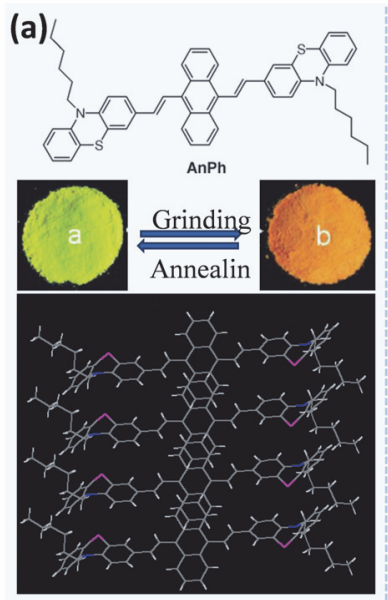

(b)

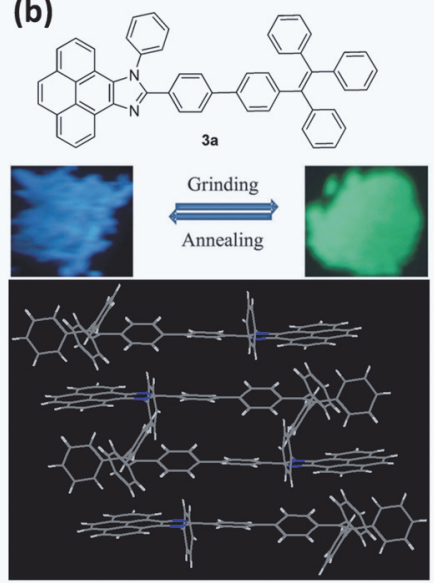

图 1 化合物 $\mathrm{AnPh}^{[26]}$ 和 $\mathbf{3} \mathbf{a}^{[27]}$ 的结构、力致变色现象和分子排列

Figure 1 The structure, mechanochromism phenomenon and molecular packing of $\mathbf{A n P h}{ }^{[26]}$ and $\mathbf{3 a}^{[27]}$

除了采用扭曲构型的片段作为分子构筑单元外，对 平面结构的修饰和细微调节也是调控分子排列和实现 力致变色性能的有效途径. $\mathrm{Li}$ 等 ${ }^{[28-30]}$ 对萠及萠衍生物的 光学特性和分子堆积进行了系统研究(图 2). 萠衍生物 (Py-H, Py-Br, Py-Bpin)在稀溶液中表现出相似的光学 性质，但在固态下则表现出完全不同的发光性质 ${ }^{[29]}$. 其 中, 晶体中呈现 monomer-like 排列的 Py-H 和 Py-Bpin 分子表现出力致变色现象，研磨前后，分子排列由有序 的 monomer-like 排列转变为无序的 dimer-like 排列, 光 谱发生红移. 并且, Py-Bpin 和 Py-Br 表现出不同的力致 发光性质, 其中, 在力刺激下, Py-Bpin 表现出力致 monomer-excimer 双发射性质，这些都与聚集态分子在 力刺激作用以后，分子排列发生变化所致. 随后，他们 还通过电子效应和位阻效应实现萠衍生物分子排列的 部分调控, 实现了高效 off-on 力致变色 ${ }^{[30]}$, 结合高压光 谱测试, 提出分子 PPCHO 的 off-on 力致变色机理归因 于分子排列发生改变, 即研磨前后分子排列由 monomer-like 排列向 dimer-like 排列转换.

分子中烷基链的长度不仅会影响分子的溶解性从 而影响分子的结晶度，而且会影响聚集态分子的排列， 进而影响发光性能 ${ }^{[31-33]} .2013$ 年, Chi 等 ${ }^{[34]}$ 通过调节烷基 链的长度得到一系列具 $\mathrm{AIE}$ 性能的分子 DSAn $(n=7 \sim$ 12) (图 $3 \mathrm{a}$ ), 研究发现, 含较短烷基链的三个分子 


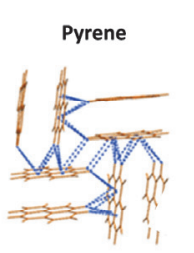

overlap (69.2\%) dimer-like packing

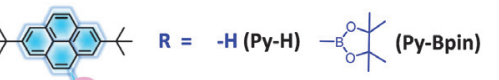

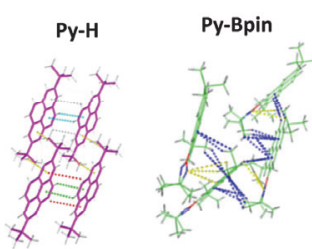

overlap (very weak) monomer-like packing

(Py-Bpin)

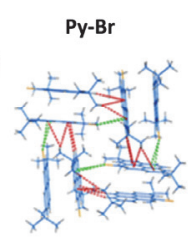

overlap (76.9\%) dimer-like packing
$\mathrm{PPOCH}_{3}$
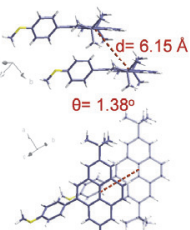

no overlap

$\mathrm{CH}_{3}\left(\mathbf{P P O C H}_{3}\right)$
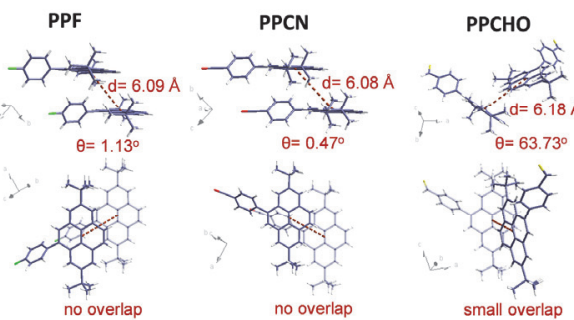

$\mathrm{PPCF}_{3}$
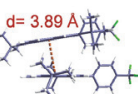

$\theta=9.98^{\circ}$
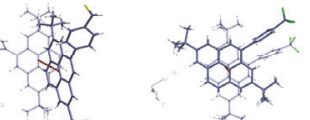

small overlap

big overlap

-Br (Py-Br)

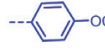

(PPF)

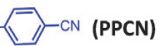

$\mathrm{CF}_{3} \quad\left(\mathbf{P P C F}_{3}\right)$

图 2 芘衍生物的分子结构和排列[29-30]

Figure 2 The structure and molecular packing of pyrene derivatives ${ }^{[29-30]}$

DSA7、DSA8 和 DSA9 二面角较小, 分子共轭较好, 在 形成聚集体时, 具有较强的分子间 $\mathrm{C}-\mathrm{H} \cdots \pi$ 相互作用(分 别有 4, 5, 4 种类型), 这种较强的相互作用不仅使分子 排列更加紧密, 同时, 分子在晶格中也更稳定. 而长烷 基链的引入导致 DSA10、DSA11 和 DSA12 的分子构象 更加扭曲，并且减弱了分子间相互作用(分别有 $1,2,2$ 种类型), 从而引起松散的分子堆积和较低的晶格能, 在外力刺激时，晶体易于破坏，这是分子 DSA10、

DSA11 和 DSA12 表现出更显著的力致变色的主要原因. Yang 等[35]通过调节芴基上的烷基链长度(图 3b), 得到 了同样的规律: 拥有较长的烷基链的分子表现出更显著 的力致变色现象.
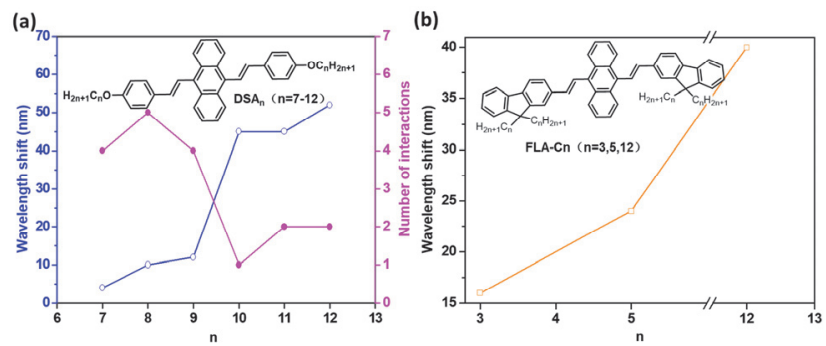

图 3 (a) 烷基链长度对力致变色性能的影响 ${ }^{[34]}$; (b) 烷基链长度对发 光性能的影响[35]

Figure 3 (a) Influence of alkyl chain length on mechanochromic properties $^{[34]}$; (b) influence of alkyl chain length on emission ${ }^{[35]}$

为了进一步研究分子堆积模式和发光行为之间的 联系, 一系列具备多晶型并伴随不同光学性质的化合物 被报道 ${ }^{[36-38]}$. 2012 年, Tian 等 ${ }^{[39]}$ 通过测试化合物 BP2VA 在不同压力下的发光光谱, 定量分析了该化合物的压致 变色过程, 并通过同质多晶现象阐述了分子排列对分子 发光性能的影响(图 4). BP2VA 在研磨前后, 发光颜色由 绿光 $\left(\lambda_{\max }=528 \mathrm{~nm}\right)$ 转变为黄光 $\left(\lambda_{\max }=561 \mathrm{~nm}\right)$, 通过加 热到 $160{ }^{\circ} \mathrm{C}$ 以上, 又可以恢复至研磨前的状态. 通过高 压光谱测试发现, 随着压力由 $0 \mathrm{GPa}$ 逐渐增加到 7.92 $\mathrm{GPa}$, 光谱由绿色 $\left(\lambda_{\max }=528 \mathrm{~nm}\right)$ 红移至红色 $\left(\lambda_{\max }=652\right.$ $\mathrm{nm})$, 当施加压力为 $2.43 \mathrm{GPa}$ 时, 发射光谱与研磨后的 粉末发射光谱非常相似. 由此可见, 在施加外部压力条 件下, BP2VA 的发光颜色发生很大变化, 这是外部压力
使分子聚集状态发生变化的结果. 为了进一步准确地研 究分子聚集状态与发光特性之间的关系，他们通过溶剂 扩散法培养单晶, 得到了 3 种不同排列模式的晶体结构 $(\mathrm{C} 1, \mathrm{C} 2$ 和 $\mathrm{C} 3)$. 在晶体 $\mathrm{C} 1$ 中, 分子沿 $y$ 轴形成 $\mathrm{J}$ 聚集的 堆积模式，无明显的 $\pi \cdots \pi$ 相互作用，晶体发射绿色苂光 $\left(\lambda_{\max }=527 \mathrm{~nm}\right)$; 在晶体 $\mathrm{C} 2$ 中, 分子 $x$ 轴发生 $\mathrm{H}$ 型聚集, 相邻的葸平面相互重叠约 $40 \%$, 面与面之间的距离约 $3.65 \AA, \pi \cdots \pi$ 相互作用较弱, 晶体发射橙色苂光 $\left(\lambda_{\text {max }}=\right.$ $579 \mathrm{~nm})$; 而在晶体 C3 中, 分子沿 $x$ 轴形成紧密的面面 堆积二聚体，面与面之间的距离约 $3.52 \AA$, 分子间具有 较强的 $\pi \cdots \pi$ 相互作用，晶体发射红色荧光 $\left(\lambda_{\text {max }}=618\right.$ $\mathrm{nm})$. 结合以上研究结果分析表明：聚集态分子的不同 排列是产生不同发光现象的主要原因. 研磨或压力作用 下使分子聚集状态发生改变, 晶体堆积模式改变从而导 致苂光颜色的变化.
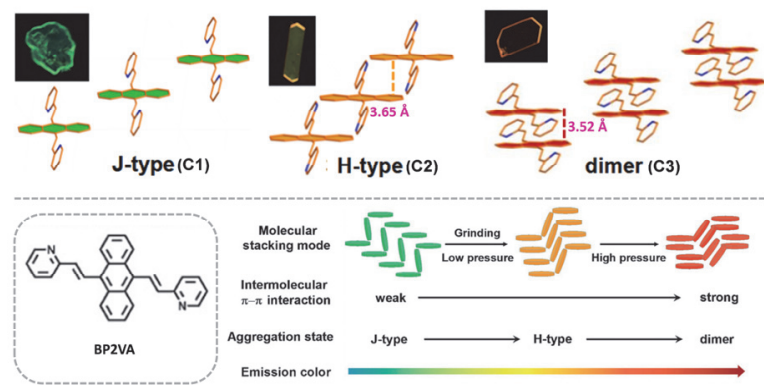

图 4 化合物 BP2VA 的分子结构、堆积模式以及不同聚集体的荧光 发射 ${ }^{[39]}$

Figure 4 The molecular structure, stacking mode and emission of different aggregates of compound $\mathbf{B P 2} \mathbf{V} \mathbf{A}^{[39]}$

2018 年, Lee 等 ${ }^{[40]}$ 报道了 PTZ-AQ 的 5 种不同的聚 集状态及其对应光学性质(图 5a), 并培养出 3 种不同晶 型(分别为晶体 $Y, O, R$ ). 研究发现, 在晶体 $Y$ 中, 相邻 分子中两个葱醌环呈平行排列，但是两个吩噻嗪呈反向 排列, 平面的重叠程度约为 $40 \%$, 平面间的距离约为 $3.662 \AA$ 和 $3.769 \AA$. 在晶体 $\mathrm{O}$ 中, 相邻分子呈头尾堆积 模式, 平面重叠程度约为 $67 \%$, 平面间的距离约为 $3.619 \AA$, 与晶体 $\mathrm{Y}$ 相比, 晶体 $\mathrm{O}$ 表现出更强的 $\pi \cdots \pi$ 相互 
作用, 光谱发射红移. 而在晶体 $\mathrm{R}$ 中, 分子呈面面堆积 模式, 并且相邻分子有两种不同的 $\pi$ 平面重叠方式, $\pi \cdots \pi$ 相互作用更强, 表现出红光发射, 并且 $\mathrm{R}$ 晶体中这种排 列有利于抑制分子无辐射运动, 提高量子产率. 值得注 意的是，分子排列不同不仅会影响苂光发射颜色的改 变, 而且, 分子的聚集状态还对材料的 TADF (thermally activated delayed fluorescence, 热活化延迟苂光)特性有 重要影响, 其中, $\mathrm{R}$ 晶体表现出高亮度的 TADF 性质.

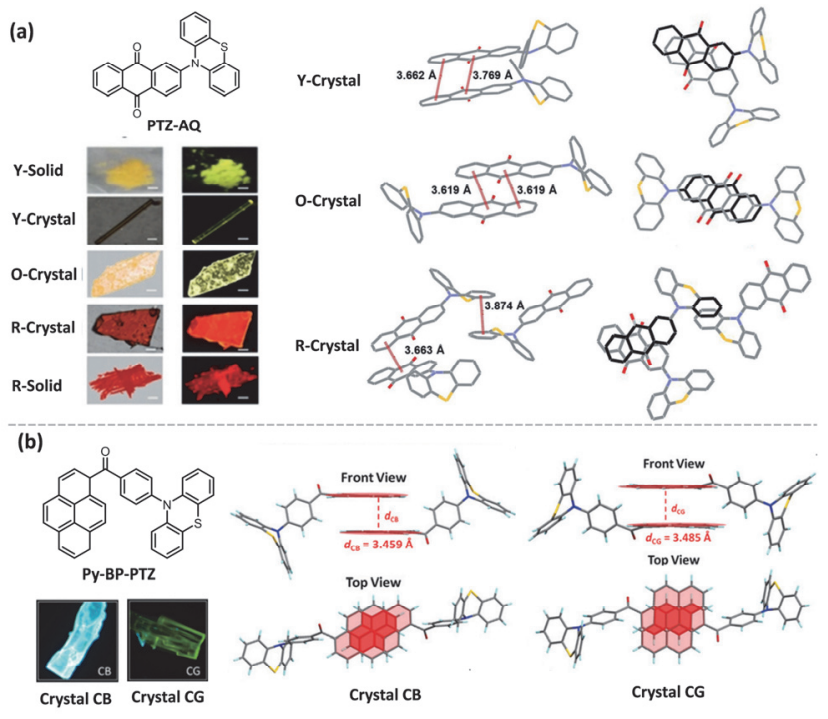

图 5 PTZ-AQ ${ }^{[40]}$ (a)和 Py-BP-PTZ ${ }^{[4]]}$ (b)的分子结构、不同聚集体的 荧光发射及堆积模式

Figure 5 The molecular structure, fluorescence emission of different aggregates and stacking modes of compound PTZ-AQ $\mathbf{Q}^{[40]}$ (a) and Py-BP-PTZ ${ }^{[41]}$ (b)

2020 年, Zhao 等 ${ }^{[41]}$ 研究了化合物 Py-BP-PTZ 的两 种晶型(CB 和 $\mathrm{CG}$ ) 排列对发光性能的影响(图 $5 b$ ), 发现 “鱼骨形”排列的晶型 CG 排列更加紧密, 分子构象更加 平面, 晶体 $\mathrm{CG}\left(\lambda_{\max }=557 \mathrm{~nm}\right)$ 体现出比 $\mathrm{CB}\left(\lambda_{\max }=463\right.$ $\mathrm{nm}$ )更加红移的光谱发射. 同时, 他们还测试了晶体 $\mathrm{CB}$ 在不同压力下的发射光谱, 随着压力逐渐增加, 分子逐 渐趋于平面化, 分子排列更加紧密, 光谱发生红移, 当 压力增加到一定程度时, 分子间强的 $\pi \cdots \pi$ 相互作用使发 射光谱的强度明显降低, 再次证明了材料的发光性质与 聚集态分子的堆积模式密切相关.

\section{3 分子排列对力致发光的影响}

力致发光 (Mechanoluminescence, ML) 是指材料在 摩擦、拉伸、压缩、冲击等力刺激作用下产生明亮发光 的现象, 早在 1605 年, 这种现象首次被 France Bacon 发 现并报道, 然而, 受限于检测条件和人们对力致发光的 认识, 有机力致发光材料种类仍然较少, 经过科学家的 坚持和努力, 最近几年, 对力致发光的研究逐渐深 $\lambda^{[42-44]}$. 在 2015 年, $\mathrm{Chi}$ 等 ${ }^{[45]}$ 将聚集诱导发光与力致发 光相结合, 这为开发高效的力致发光材料提供了新的思
路.

扭曲构型的分子间相互作用和抑制分子间 $\pi \cdots \pi$ 堆积 有助于构建高亮度力致发光材料, 四(三)苯乙烯(TPE) 由于其特殊的扭曲构型被广泛应用于力致发光材料领 域 ${ }^{[46-54]}$. 为了研究分子排列对力致发光的影响, $\mathrm{Li}$ 等报 道了一系列具有力致发光性质的有机小分子化合物, 对 分子结构-排列-性能进行了系统、深入的研究. 2016 年, 他们 ${ }^{[46]}$ 报道了一种多晶型化合物 TMPE(图 6a), 其中 $\mathrm{C}_{\mathrm{p}}$ 型晶体中强的相互作用使分子堆积更加紧密, 在很大程 度上降低了分子非辐射跃迁带来的能量损失, 表现出力 致发光性能. 而 $\mathrm{C}_{\mathrm{c}}$ 型晶体中分子间相互作用较弱, 分子 排列较松散, 无 ML 现象. 同时, 他们还通过改变 TMPE 分子上烷基链的长度调控分子排列, 当烷基链增 长时, 分子 TBPE 和 THPE 的排列变得疏松, 分子间相 互作用减弱, 无 ML 现象产生. 另外, 他们还报道了一 种纯碳氢 ML 材料 TETPE 和一系列基于 TPE 的同分异 构体 ${ }^{[47-48]}$, 研究结果证明, 分子间强的相互作用和紧密 的分子堆积是产生 ML 的主要原因. 该结论也在分子 $m m-\mathrm{TPE}(\mathrm{PI})_{2}$ 和 $p p$-TPE $(\mathrm{PI})_{2}$ 中进一步被证实 ${ }^{[49]}$. Chi 等 ${ }^{[50]}$ 也研究了基于 TPE 的 ML 材料, 研究发现，与平行 或反平行排列模式的分子 $\boldsymbol{p}$-P4Ac 和 $\boldsymbol{p}$-FP2A 相比, 非平 行排列模式的分子 $\boldsymbol{p}$-P4A 更有利于形成非中心对称排 列, 有助于 ML 的产生. 并且, 他们研究了 P4TA 的两种 晶型 $\mathrm{SC}_{\mathrm{g}}$ 和 $\mathrm{SC}_{\mathrm{b}}$ 的不同 $\mathrm{ML}$ 活性(图 $6 \mathrm{~b}$ ), 并指出不同性 能的产生归因于它们不同的分子堆积模式 ${ }^{[45]}$. 李冰石 课题组 ${ }^{[51-52]}$ 也报道了一系列基于 TPE 的 ML 材料, 并对 分子间相互作用力和分子排列模式对 ML 的影响进行了 讨论.

随后, $\mathrm{Li}$ 等 ${ }^{[53}$ 将 $\mathrm{AIE}$ 特性与自组装性能相结合, 设 计并合成了基于三苯乙烯的力致发光分子 tPE-5-Meth (图 6c), 研究发现, 在 Crystal-P1 中, 相邻的分子具有更 强的分子间/分子内相互作用，这种强的分子间相互作 用有助于形成有效的网状结构, 从而形成更加紧密的空 间堆积, 体现出 ML 性能. 而 Crystal-P2 相邻的分子相互 呈反平行排列, 分子间作用力较弱, 无 ML 现象产生. 并且, Crystal-P1 通过简单的热处理便可以实现力致发 光性能的循环. 利用噻吩环这种具有自组装效应的功能 基团, 他们 ${ }^{[5]}$ 研究了具有自组装能力的力致发光材料 (图 6d), 与中心对称的反平行堆积的 tPE-Ph 相比, 其中 tPE-2-Th 和 tPE-3-Th 中由于自组装单元的存在, 呈现 出非中心对称的紧密的鱼骨状堆积方式, 这有利于高亮 度的力致发光性能的产生，同时，利用三明治结构的柔 性器件成功建立了压力大小和发光强度之间的定量关 系，制备了灵活的可穿戴设备，并在通信、信息存储以 及医疗监护方面表现出潜在的应用.

具有扭曲 “蝴蝶状” 构型的吩噻嗪也是构筑 ML 材 料的常用构筑单元, 2015 年, Chi 等 ${ }^{[55]}$ 报道了不对称结构 化合物 $\mathbf{1}$ 的力致发光性质，在晶体排列中，化合物 $\mathbf{1}$ 表 
(a)
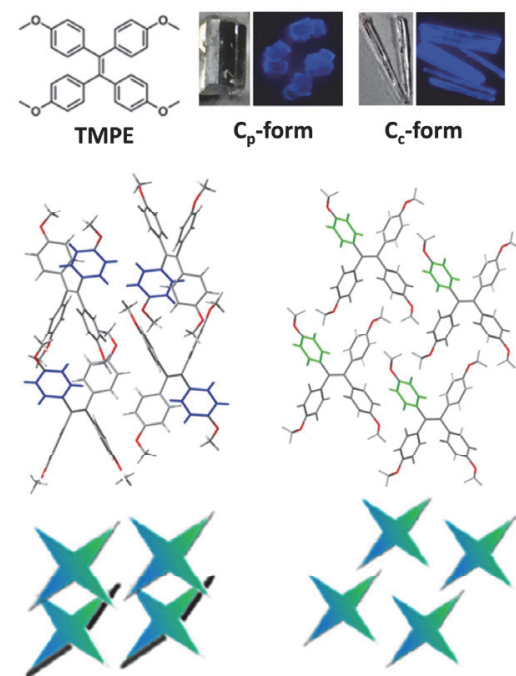

$\mathrm{C}_{\mathrm{p}}$-form (ML active)

$\mathrm{C}_{\mathrm{c}}$-form (ML inactive) (b)
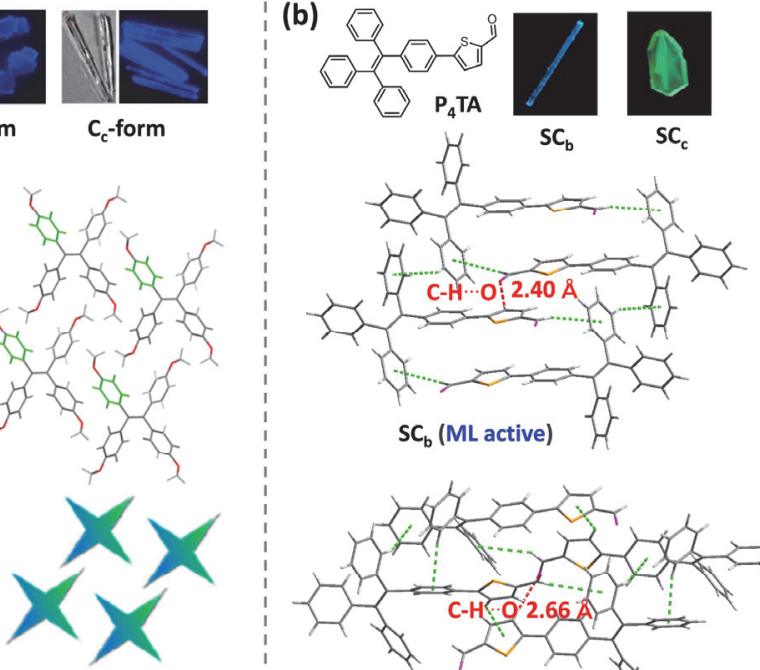

(d)

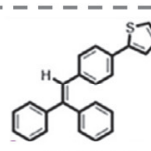

tPE-2-Th

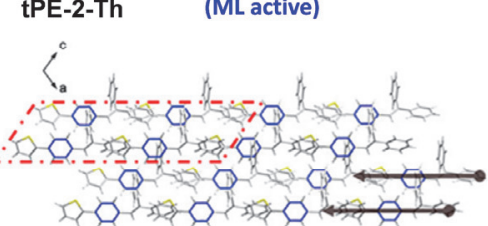

'Parallel in the same direction!

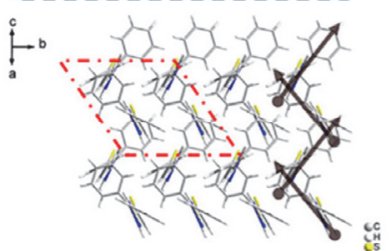

'Fishbone structure, Noncentrosymmetry'

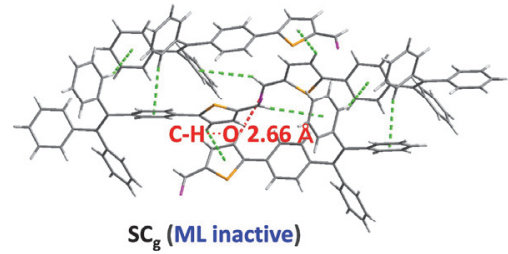

$\mathrm{SC}_{\mathrm{g}}$ (ML inactive)

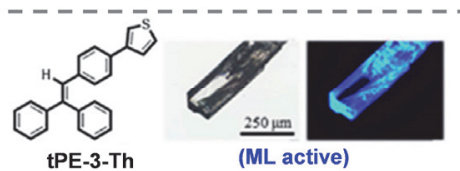

tPE-3-Th (ML active)

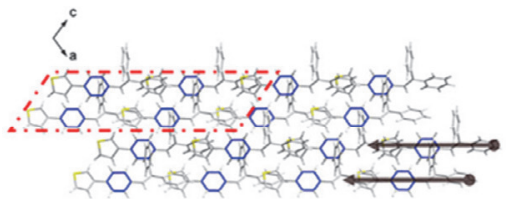

'PParallel in the same direction! c:

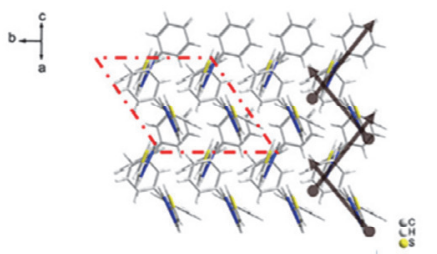

'E-ishbone structure, Noncentrosymmetry (c)

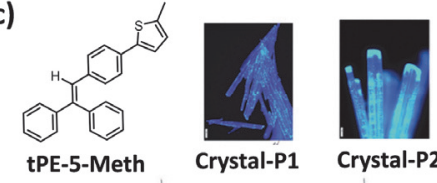

tPE-5-Meth Crystal-P1 Crystal-P

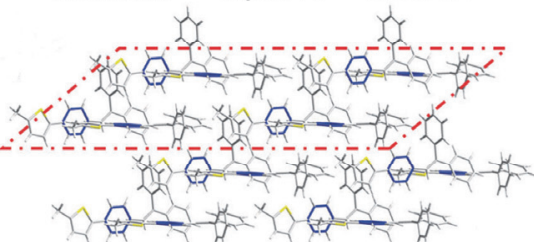

Crystal-P1 (ML active)

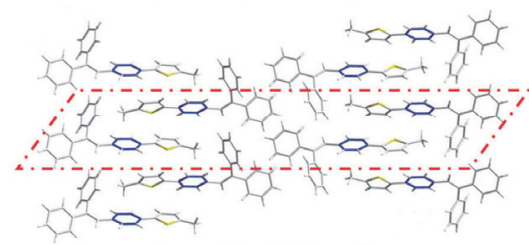

Crystal-P2 (ML inactive)

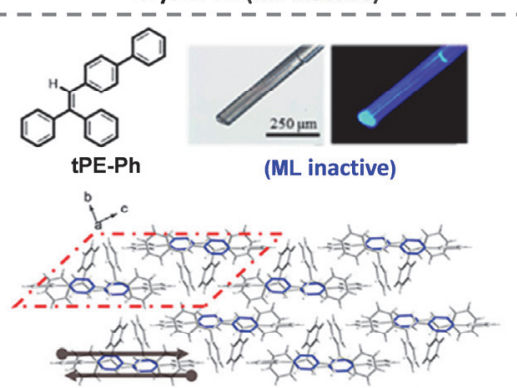

'Parailet in the opposite direction!

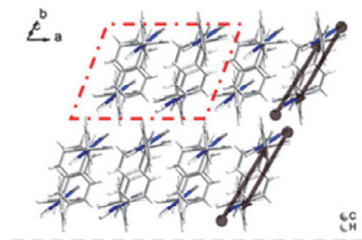

'Ántiparallel structure, Centrosymmetry!

图 $6(\mathrm{a} \sim \mathrm{c})$ 基于四(三)苯乙烯衍生物的同质多晶、分子排列和相应的力致发光性能 ${ }^{[46,50,53]}$; (d) 三苯乙烯衍生物分子结构、排列和力致发光性能 ${ }^{[54]}$ Figure $6 \quad(\mathrm{a} \sim \mathrm{c})$ Polymorphism, molecular packing and corresponding mechanoluminescence properties of tetra(tri)styrene derivatives ${ }^{[46,50,53]}$; (d) molecular structure, packing and mechanoluminescence properties of tristyrene derivatives ${ }^{[54]}$

现出微妙的分子堆积, 相邻分子形成如中国 “八卦”一 样的排列模式(图 7a), 其中每个分子的平面咔唑部分与 另一个分子的非平面吩噻嗪部分互锁形成紧密有序的 排列，分子间无 $\pi \cdots \pi$ 相互作用. 相比之下，分子 2 虽然无 分子间 $\pi \cdots \pi$ 相互作用，但表现出松散的堆积模式，且分 子相互作用较弱, 在力的刺激下易产生无辐射跃迁. 分 子 3 的排列中观察到明显的 $\pi \cdots \pi$ 相互作用, 表现出聚集 诱导淬灭 (ACQ)效应, 因此, 分子 $\mathbf{1}$ 的力致发光性能与 其聚集态分子的排列密切相关.

2017 年, $\mathrm{Li}$ 等 ${ }^{[56]}$ 报道了首例同时具备 ML 和室温磷 光的吩噻嗪衍生物 $\mathrm{CzS}_{-} \mathrm{CH}_{3}$ 和 $\mathrm{CzS}_{-}-\mathrm{C}_{2} \mathrm{H}_{5}$ (图 7b), 在晶 体中存在有效的分子间氢键相互作用, 包括 $\mathrm{C}-\mathrm{H} \cdots \mathrm{N}$ 和
$\mathrm{C}-\mathrm{H}^{\cdots} \pi$ 键, 但无苯环间的 $\pi \cdots \pi$ 堆积. 相邻的两个分子 通过多重的 $C-\mathrm{H}^{\cdots} \cdots \pi$ 和 $\mathrm{C}-\mathrm{H} \cdots \mathrm{N}$ 键相互作用形成了二聚 体, 然后再由二聚体的规整排列组成单晶, 这些强的分 子间相互作用能够在很大程度上减小机械力刺激下的 分子滑移和无辐射运动导致的能量损失, 有利于 ML 现 象的产生. 2018 年, 他们 ${ }^{[57]}$ 还报道了颜色可变的 ML 分 子 FCOCzS, 在连续的机械刺激下, 其动态机械发光从 蓝色到白色再到黄色，这种独特的动态 ML 效应可归因 于机械刺激下分子构象的改变. 分子间较强的相互作用 限制晶体在机械刺激下的佣塌和滑移，并使其具有良好 的机械稳定性. 


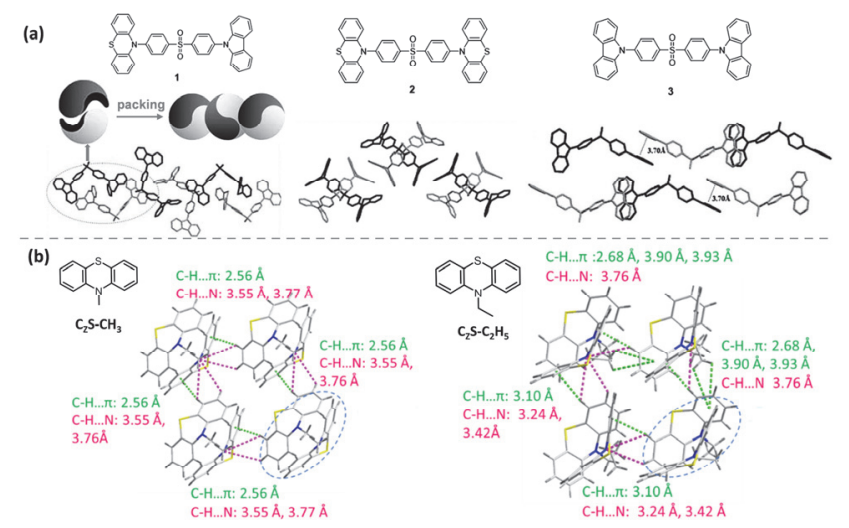

图 7 吩噻嗪衍生物的分子排列和力致发光 ${ }^{[55-56]}$

Figure 7 Molecular packing and mechanoluminescence properties of phenothiazine derivatives ${ }^{[55-56]}$

2019年, Xu等 ${ }^{[58]}$ 以吩噻嗪为给体, 研究了一对手性 对映异构体及其外消旋体，他们不仅表现出明显不同的 圆偏振发光行为, 而且表现出完全相反的 ML 活性. 单 晶数据表明, 对映异构体的 ML 可能被其分子手性激活, 使其具有极性空间群，并且，对映异构体分子间的作用 力较强, 分子堆积较为紧密, 而外消旋体的分子间氢键 明显减少, 分子堆积疏松. 这些实验结果再一次证明了 ML 与分子排列密切相关. Thilagar 和 Ghosh 等 ${ }^{[59-60]}$ 也报 道了非中心对称吩噻嗪硼烷衍生物的 ML 性能.

具有独特电子特性和空间构型的三苯胺(TPA)也是 常用的 ML 分子构筑单元. 2017 年, $\mathrm{Li}$ 等 ${ }^{[61]}$ 将醛基和三 苯胺结合, 得到了具有 ML 性质的分子 TPA-1BA, 研究 表明, 三苯胺的扭曲构型有利于抑制分子间 $\pi \cdots \pi$ 相互作 用, TPA-1BA 的晶体排列中, 较强的相互作用力增强了 该晶体的刚性, 有助于抑制分子滑移造成的无辐射跃 迁, 促进 ML 的产生. 2018 年, 他们在三苯胺上引入不 同卤素 ${ }^{[62]}$, 调节分子间相互作用, 研究 ML 性能和分子 排列之间的关系, 发现均匀分布的分子间较强的相互作 用导致分子堆积更加紧密, 从而增加了晶体刚性, 降低 分子在研磨或刮擦时的滑移和塌陷而造成的无辐射跃 迁, 有利于产生力致发光. 但分子间相互作用和晶体刚 性太强, 不利于 ML 的产生, 因此, 合适的分子间作用 力和晶体刚性是产生 ML 的必要条件. 2019 年, 他们再 次证明卤素的引入有助于增强分子间相互作用 ${ }^{[63]}$, 从 而增加分子的刚性，促进 ML 的产生.

最近, $\mathrm{Li}$ 课题组报道了 ${ }^{[64]}$ 一种同质多晶的 TPA 衍生 物 TPA-o-3COOMe(图 8a), 它不仅同时具备 ML 和 RTP 两种特性, 而且两种不同的性能分别承载在两种晶型 里, 其中, 晶体 $\mathrm{A}$ (中心对称排列)表现出肉眼可见的室 温磷光性质, 晶体 $\mathrm{B}$ (非中心对称排列)则表现出 $\mathrm{ML}$ 性 能. 通过研究不同排列与不同性能间的关系, 发现呈现 反平行的均一排列的晶体 A 中, 相邻分子间相互作用较 强, 形成了二聚体, 有利于室温磷光的产生, 而形成无 序的空间立体网状排列的晶体 $\mathrm{B}$, 则有利于抑制力刺激
作用下分子滑移造成的能量损失, 促进 ML 的产生. 这 一现象充分说明了发光性能与分子排列间的依赖关系.

除了常见的扭曲结构以外, 苯并咪唑衍生物也体现 出 ML 性能 ${ }^{[65-66]}$. Li 课题组 ${ }^{[65]}$ 报道了化合物 tPTI-Bpin 的多晶型和不同的性能(图 8b), 晶体 tPTI-B1 呈现出稳 定的 “三角形” 排列, 在力刺激下能有效抑制滑移带来 的能量损失, 表现出 ML 现象. 而晶体 tPTI-B2 中, 相邻 分子反平行成对排列, 体现出 “平行四边形” 的排列效 果, 力刺激下, 分子易滑移, 促进无辐射跃迁, 无 ML 现 象产生. Xia 等 ${ }^{[61]}$ 也报道了具有同质多晶现象的咪唑衍 生物 TBIM, 两种晶型 TBIMB 和 TBIMG 都表现出明 显的 ML.

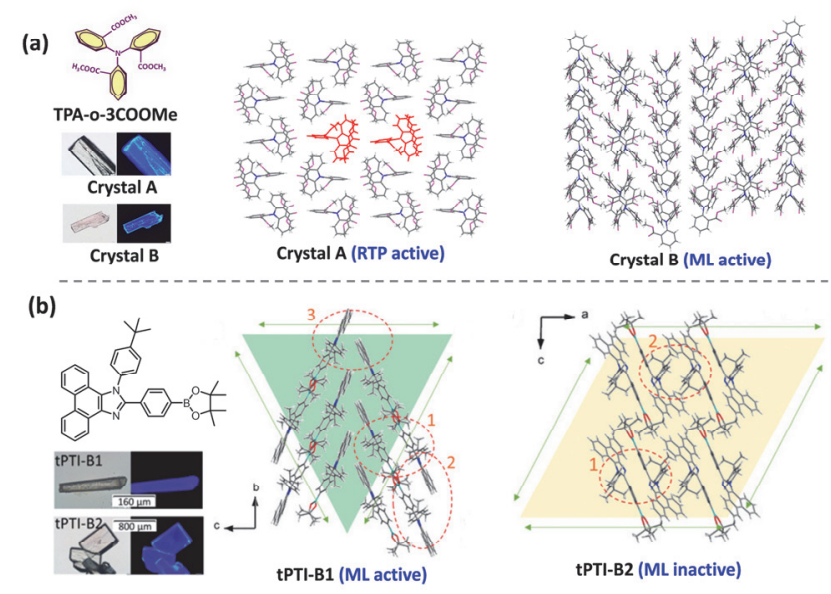

图 8 (a) TPA-o-3COOMe 的同质多晶形、分子排列及不同的发光性 能 ${ }^{[64]} ;$ (b) 咪唑衍生物的同质多晶形、分子排列及不同的力致发光性 能 ${ }^{[65]}$

Figure 8 (a) Polymorph, molecular packing and different luminescence properties of TPA-o-3COOMe ${ }^{[64]}$; (b) polymorphism, molecular packing and different mechanoluminescence properties of imidazole derivatives ${ }^{[65]}$

一些咔唑类衍生物也可以通过调节分子排列而表 现出 ML 现象. Chi 等 ${ }^{[67}$ 利用烷基链的自组装效应, 通过 调节咔唑上烷基链的长度调控分子排列和分子间相互 作用, 得到了具有 ML 性能的 Cz-alkyl-6 和 Cz-alkyl-7. 在晶体排列中, 两种化合物中的烷基链以结晶状态聚集 并堆叠在一起, 形成烷基链区，在该区域内分子间的相 互作用较弱, Cz-alkyl-6 晶体中 6 个分子聚集在一起形 成锥形漏斗状排列, Cz-alkyl-7 中烷基链沿着咔唑呈现 螺旋状排列. 晶体中的烷基链区和松散堆积导致 ML 的 低压阈值, 有利于提高材料的 ML 敏感度. 同时, 他们 将 Cz-alkyl-6 与不同发光颜色的荧光染料掺杂, 实现了 ML 颜色的可调性，该方法同时也被 Yang 等 $\left.{ }^{[6]}\right]$ 报道.

除了上述的力致苂光外, 有些特殊化合物还可表现 出力致荧光和磷光双发射的性质. $\mathrm{Li}$ 等 ${ }^{[69]}$ 首次报道了力 致荧光和磷光双发射的分子 DPP-BO, 由于 DPP-BO 螺 旋桨状的分子构型，在晶体中无 $\pi \cdots \pi$ 堆积，但分子间相 互作用较强, 能有效抑制机械力刺激下的无辐射运动, 
有助于实现高亮度的 ML. 随后, 他们 ${ }^{[70]}$ 通过引入重原 子增强自旋轨道耦合得到的化合物 BrFlu-CBr，在紫外 光激发或者力刺激下表现苂光-磷光双发射性质. 长时 间的机械力刺激作用后, BrFlu-CBr 样品的力致发光颜 色由天蓝色转变为蓝色, 这主要是由于化合物的晶体堆 积受到了一定程度的破坏, 导致力致磷光效率下降所 致. $\mathrm{Xu}$ 等 ${ }^{[71]}$ 报道了化合物 $\mathbf{I m B r}$, 不仅有长寿命的光致 发光, 也有着长寿命的室温力致发光. 这种力致长余辉 发光现象与溴原子导致的强的自旋轨道耦合作用有关, 同时也与排列中 $\mathrm{H}$ 聚集的形成和非中心对称晶体结构 对分子内运动的限制密切相关.

由此可见, 力致发光与分子堆积密切相关, 在力致 发光分子中, 分子排列大体表现出紧密的堆积方式, 有 效抑制了分子间 $\pi \cdots \pi$ 相互作用对发光的猝灭效应，同时， 通过大量的分子间相互作用抑制了分子运动所带来的 非辐射跃迁. 而且, 此类排列方式所产生的类似分子间 互锁结构, 对力刺激下聚集态结构的稳定性起到了巩固 和加强的作用, 有效减少了分子滑移所带来的能量损 失, 促进高亮度力致发光现象的产生.

\section{4 分子排列对室温磷光的影响}

室温磷光(RTP)材料因其独特的发光性质和应用前 景而受到广泛关注 ${ }^{[72-79]}$. 近几年, 纯有机 RTP 材料的开 发和研究取得了一定的进展. 研究者发现, 室温磷光性 能不仅与分子结构有关, 而且, 还与分子堆积方式及分 子间的相互作用密切相关 ${ }^{[74]}$.

2010 年, 唐本忠等 ${ }^{[80]}$ 首次提出了结晶诱导室温磷 光的理念, 报道了一系列二苯酮及其衍生物在结晶时表 现出室温磷光的现象. 研究发现, 分子在结晶过程中发 生长程有序的分子堆积和排列, 两个相邻分子之间通过 较强的氢键相互作用, 这种较强的分子间相互作用有助 于稳定分子构象, 从而减少了分子运动和碰撞带来的能 量损失 ${ }^{[81]} .2015$ 年, 安众福等 ${ }^{[82]}$ 提出了一种获得长寿命 室温磷光的策略. 他们通过分子的结构调节和简单的基 团修饰一方面促进分子的 $n-\pi$ * 跃迁和单线态到三线态 的系间窝跃; 另一方面促使分子形成稳定的 $\mathrm{H}$ 聚集排列 模式, 增强分子间耦合作用从而获得长寿命室温磷光. 因此，聚集态分子间较强的相互作用能有效抑制非辐射 跃迁和稳定三线态激子, 这也是目前已报道的有机室温 磷光分子大都基于晶体结构的重要原因.

$\mathrm{Li}$ 等 ${ }^{[83]}$ 研究了一系列嗍酸(酯)衍生物, 结合分子排 列和性能分析发现, 通过分子间强相互作用可以抑制分 子运动, 降低非辐射跃迁带来的能量损失, 分子间形成 有效的 $\pi \cdots \pi$ 堆积有利于稳定激发三线态, PBA-MeO 的 寿命长达 $2.24 \mathrm{~s}$. 他们还将二苯酮与硼酸酯相结合, 报 道了一系列具有室温磷光的化合物 ${ }^{[84]}$, 其中, 由于分子 排列的不同, 具有同质多晶现象的 BP-p-BO 表现出不 同的磷光寿命.
为了深入探究分子排列对室温磷光的影响, $\mathrm{Li}$ 等 ${ }^{[85]}$ 报道了具有同质三晶的室温磷光分子 $\mathbf{C z S}-\mathbf{C N}$ (图 9a), 研究发现，同一分子结构的三种不同排列的晶体(A, B 和 $\mathrm{C}$ )分别对应不同的室温磷光性能，通过分析三种晶 体的分子排列和构象发现, 晶体 $\mathrm{A}$ 中相邻分子通过较强 的 $\pi \cdots \pi$ 相互作用和分子间氢键作用形成稳定的排列，这 些有效的分子间相互作用能够抑制分子运动产生的无 辐射跃迁，从而促进长寿命的室温磷光 $(266 \mathrm{~ms})$ 和高的 量子产率 $(22.6 \%)$. 而晶体 B 中, 分子间相互作用明显减 弱, 从而导致较短的室温磷光寿命 $(41 \mathrm{~ms})$ 和较低的量 子产率(17.8\%). 与晶体 $\mathrm{A}$ 和 $\mathrm{B}$ 的 $\mathrm{X}$ 型堆积方式相比, 晶 体 $\mathrm{C}$ 表现出反平行堆积方式, 并且晶体 $\mathrm{C}$ 中的分子, 激 发态的系间窝越能力较弱, 因此晶体 $\mathrm{C}$ 表现出较短的室 温磷光寿命 $(32 \mathrm{~ms})$ 和较低的发光量子产率 $(6.9 \%)$. 因 此, 分子排列对室温磷光的亮度和寿命都有重要影响. $\mathrm{Li}$ 等 ${ }^{[86]}$ 通过盐酸刺激具有 RTP 现象的吩噻嗪衍生物 CzS-o-py(图 9b), 在酸刺激前后, 分子排列由原本的紧 密堆积变为疏松的堆积, RTP 现象也随之消失, 这是由 于晶体中 $\mathrm{HCl}$ 和水分子的引入改变了 CzS-o-py 的堆积 模式, 从而呈现出不同的 RTP 性能.

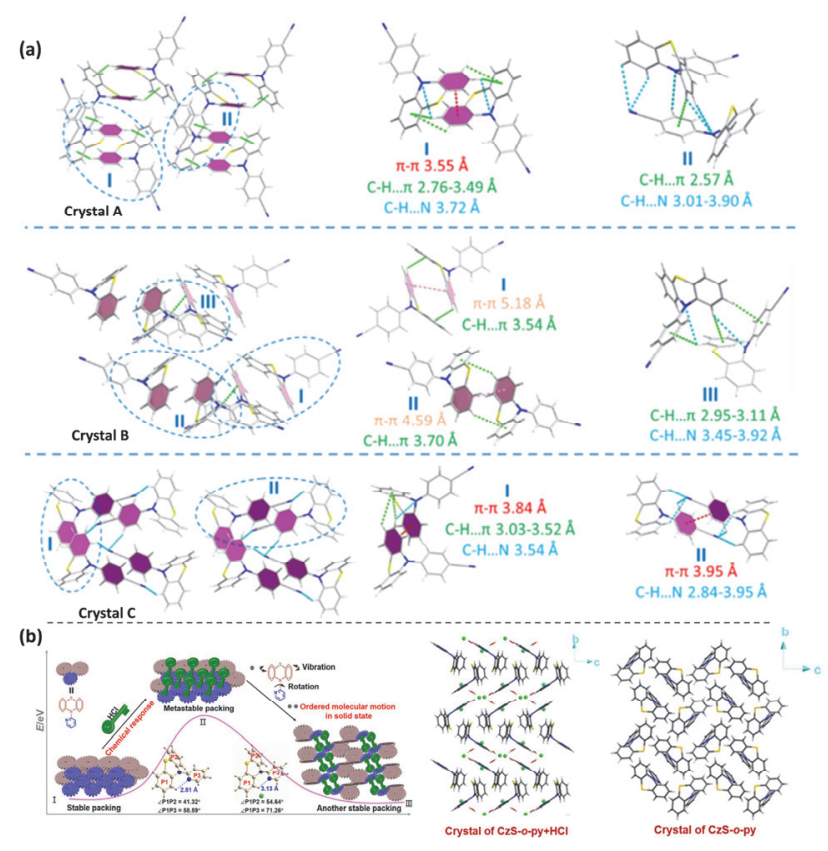

图 9 (a) 化合物 CzS-CN 的同质多晶形的室温磷光和分子排列 ${ }^{[85]}$; (b) 化合物 CzS-o-py 在酸刺激前后的室温磷光和分子排列 ${ }^{[86]}$

Figure 9 (a) RTP properties and molecular packing of three polymorphism of compound CzS-CN ${ }^{[8]}$; (b) RTP properties and molecular packing of compound CzS-o-py before and after acid stimulation ${ }^{[86]}$

另外，通过结构的细微调节可以实现不同的分子排 列和 RTP 性能. 例如，在氧化吩噻嗪上引入不同长度的 烷基链调节分子排列[87]，研究发现室温磷光的寿命体 现出明显的奇-偶效应：链长为奇数碳数的分子表现出 较长的室温磷光寿命, 其中, $\mathrm{CS}_{-} \mathrm{C}_{3} \mathrm{H}_{7}$ 的室温磷光寿命 达 $327 \mathrm{~ms}$. 通过研究分子排列发现，相邻分子间 $\pi \cdots \pi$ 重 
叠程度较大, 分子间距离较短, $\pi \cdots \pi$ 相互作用更强是 $\mathrm{CS}_{-} \mathrm{CH}_{3} 、 \mathrm{CS}-\mathrm{C}_{3} \mathrm{H}_{7}$ 和 $\mathrm{CS}-\mathrm{C}_{5} \mathrm{H}_{11}$ 体现出较长室温磷光寿 命的主要原因. 控制分子的电子特性也是调控分子在固 态下的堆积方式的有效策略(图 10a), Li 等 ${ }^{[88]}$ 研究发现, 当取代基为给电子特性的基团时, $\mathrm{CS}-\mathrm{CH}_{3} \mathrm{O}$ 和 $\mathrm{CS}-\mathrm{CH}_{3}$ 表现出微弱的分子间 $\pi \cdots \pi$ 相互作用, 磷光寿命较短, 分 别为 $88 \mathrm{~ms}$ 和 $96 \mathrm{~ms}$; 当取代基调节为氢原子时, CS-H 表现出了适中的分子间 $\pi \cdots \pi$ 相互作用, 寿命增长为 188 $\mathrm{ms}$; 随着取代基调节为拉电子基团时，化合物 CS-Br、

CS-CI 和 CS-F 在排列中具有更强的分子间 $\pi \cdots \pi$ 相互作 用, 相邻分子的两个苯环相互平行, 并且其质心距离都 较近, 分别为 $3.677 \AA 、 3.732 \AA$ 和 $3.773 \AA$, 寿命呈现出 明显的增长, 尤其是分子 CS-F, 磷光寿命长达 $410 \mathrm{~ms}$. 当取代基调整为强拉电子基团 $\left(-\mathrm{CF}_{3}\right)$ 时, 分子 $\mathbf{C S}-\mathbf{C F}_{\mathbf{3}}$ 表 现出光诱导室温磷光现象, 通过光照前后的晶体分析发 现, 光照后, 分子排列发生变化, 分子间的 $\pi \cdots \pi$ 相互作 用增强, 导致了长寿命的磷光发射. 这种光照前后引起 分子排列发生变化, 从而导致室温磷光发生改变的现象 也被分子 TPA-B 证实 ${ }^{[89]}$. 因此, 由电子特性引发的分子 堆积中强的 $\pi \cdots \pi$ 相互作用应该是稳定激发三线态, 实现 超长室温磷光寿命的主要因素, 由此, $\mathrm{Li}$ 等 ${ }^{[88]}$ 于 2018 年 提出了 MUSIC (Molecular Uniting Set Identified Characteristic)概念.

$\mathrm{Li}$ 等 ${ }^{[90]}$ 将电子效应和空间位阻效应相结合, 引入 不同体积和电子特性基团来调节聚集态分子的堆积方 式(图 10b). 当取代基为位阻较大的刚性苯环时, 相互平 行排列的二聚体间氢键较长 (2.862 ̊), 磷光寿命较短 (52 ms). 引入拉电子基团 $\mathrm{Cl}$, 分子间氢键有所增强 $(2.742 \AA)$, 磷光寿命也相应增长为 $139 \mathrm{~ms}$. 当取代基调 节为位阻较小的柔性叔丁基时, 分子间氢键进一步增强 (a)

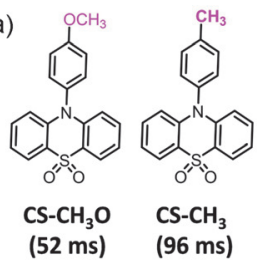

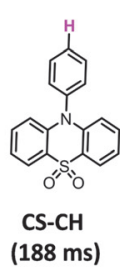

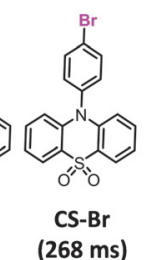

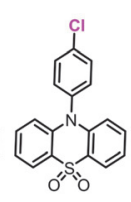

CS-Cl
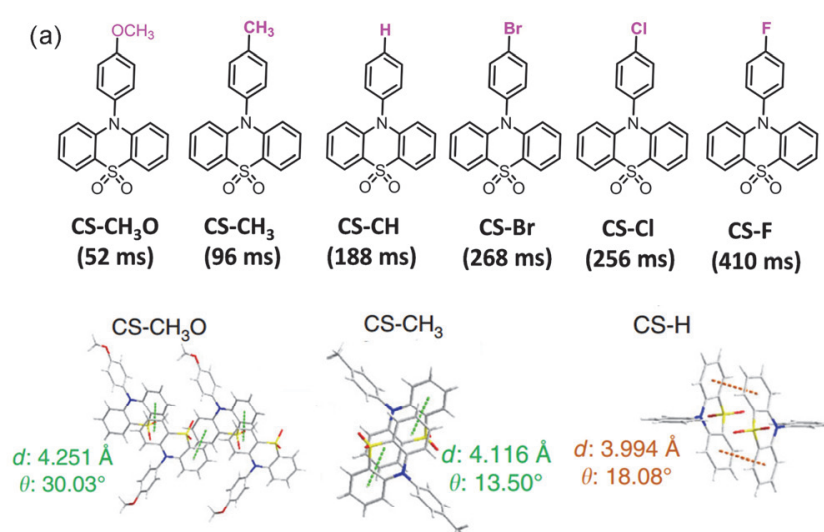

CS-H
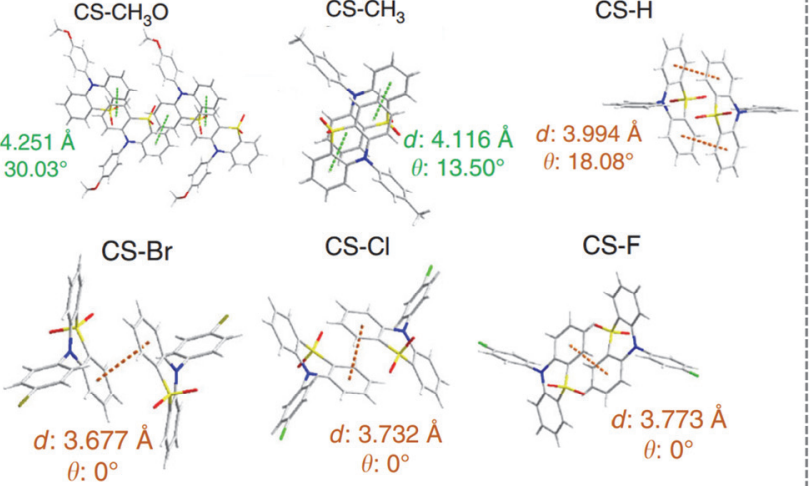

CS-Cl

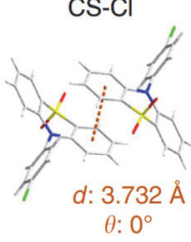

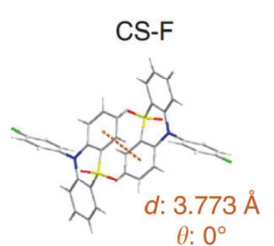

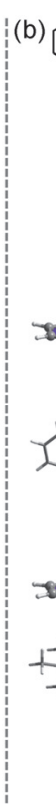

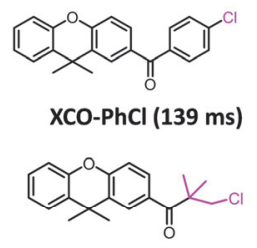

XCO-PiCl(601 ms)
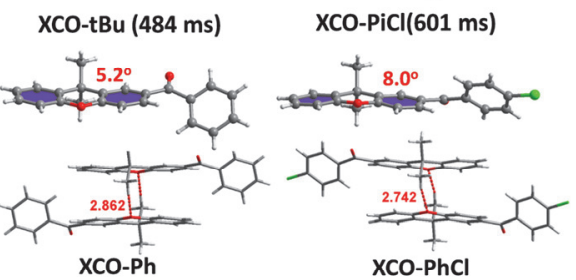

xCO-PhCl
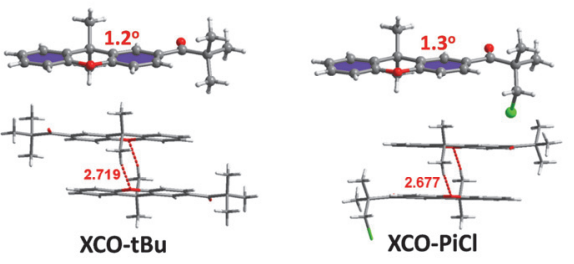

图 10 吩噻嗪衍生物的分子排列和室温磷光 ${ }^{[88,90]}$

Figure 10 Molecular packing and RTP properties of phenothiazine derivatives ${ }^{[88,90]}$ 
(a)

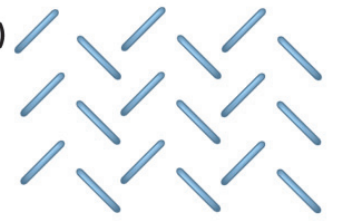

(c)

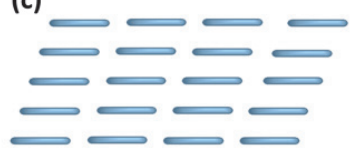

(b)

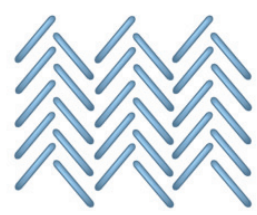

(d)

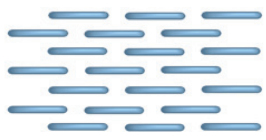

图 11 有机半导体的常见分子堆积结构[85-89]

Figure 11 Typical molecular packing motifs in organic semiconductors $^{[85-89]}$

极性基团(例如: 卤素原子)的引入可以调节分子排 列，增强 $\pi \cdots \pi$ 相互作用. 2004 年, Bao 等 ${ }^{[98]}$ 合成了一系列 卤代并四苯衍生物并对其进行性能测试和单晶分析, 研 究发现, 单取代的 5-溴和 5-氯并四苯呈鱼骨状堆积, 而 5,11 -二氯并四苯具有明显的 $\pi$ 轨道重叠. 在单晶晶体管 中, 5,11 二二氯并四苯的迁移率高达 $1.6 \mathrm{~cm}^{2} \cdot \mathrm{V}^{-1} \cdot \mathrm{s}^{-1}$. 因 此, 增加 $\pi$ 轨道重叠并促进载流子传输可能是造成高迁 移率的原因. 2015 年, Pei 等 ${ }^{[99]}$ 在共轭骨架的不同位置引 入不同数目的氟原子(图 12a), 得到的一系列化合物的

单晶排列随分子偶极矩与静电势的改变而逐渐发生变 化, 并且其器件性能结果也随其单晶排列不同而不同.

烷基链的引入也是调节分子排列常见的策略，并且 还可以调节分子的溶解性和成膜性 ${ }^{[100-101]}$. 无取代的并 五苯在晶态下表现出鱼骨状排列，当在并五苯上引入硅 烷基时, 分子间 $\pi \cdots \pi$ 重叠增强, 变为面对面排列, 这种 $\pi-\pi$ 重叠程度的增加有助于载流子的迁移 ${ }^{[102]}$.

烷基链长度的微小改变可以引起分子排列的巨大 差异, Jackson 等 ${ }^{[103]}$ 在共轭单元上引入不同长度的烷基 链(图 12b), 其中, 烷基链为异丙基和甲基时, 分子表现 出鱼骨状排列, $\pi \cdots \pi$ 相互作用弱，而烷基链为乙基时，分 子表现出明显的 $\pi \cdots \pi$ 堆积, 分子间距离为 $1.57 \AA$, 迁移 率最大, 达 $1.0 \mathrm{~cm}^{2} \cdot \mathrm{V}^{-1} \cdot \mathrm{s}^{-1}$. $\mathrm{Li}$ 等 ${ }^{[104]}$ 在荎酰亚胺衍生物 上分别引入不同长度的烷基直链和不同分叉位点的烷 基支链, 研究了烷基链对载流子传输性能的影响, 其中, DPPDI-R8 体现出高达 $1.38 \mathrm{~cm}^{2} \cdot \mathrm{V}^{-1} \cdot \mathrm{s}^{-1}$ 的迁移率, 因此, 烷基链的引入可形成自组装效应，并调节聚集态分子的 排列.

烷基链对器件性能的影响是多个因素综合互相影 响的，比如分子排列、分子结晶性、薄膜的形貌等. Pei 等 ${ }^{[105]}$ 引入不同长度烷基链将两个 FAI 单元连接起来, (a)

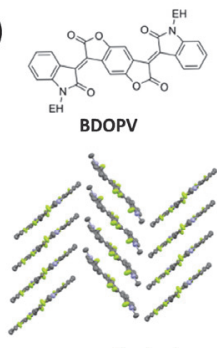

$\mu=1.90 \mathrm{~cm}^{2} \mathrm{~V}^{-1} \mathrm{~s}^{-1}$

$-1---1-1-$
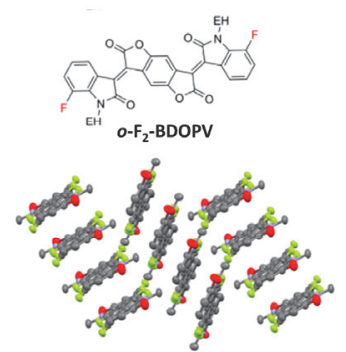

$\mu=1.63 \mathrm{~cm}^{2} \mathrm{~V}^{-1} \mathrm{~s}^{-1}$
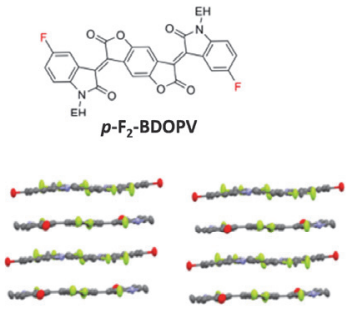

$\mu=3.86 \mathrm{~cm}^{2} \mathrm{~V}^{-1} \mathrm{~s}^{-1}$
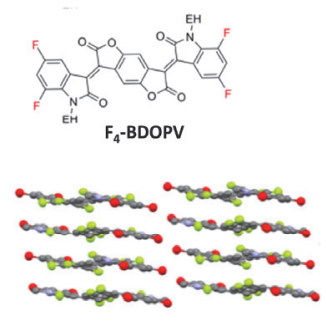

$\mu=7.58 \mathrm{~cm}^{2} \mathrm{~V}^{-1} \mathrm{~s}^{-1}$
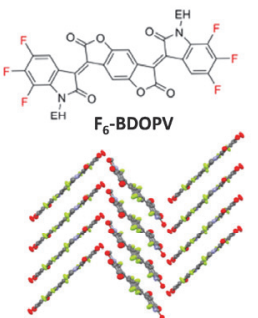

$\mu=3.25 \mathrm{~cm}^{2} \mathrm{~V}^{-1} \mathrm{~s}^{-1}$
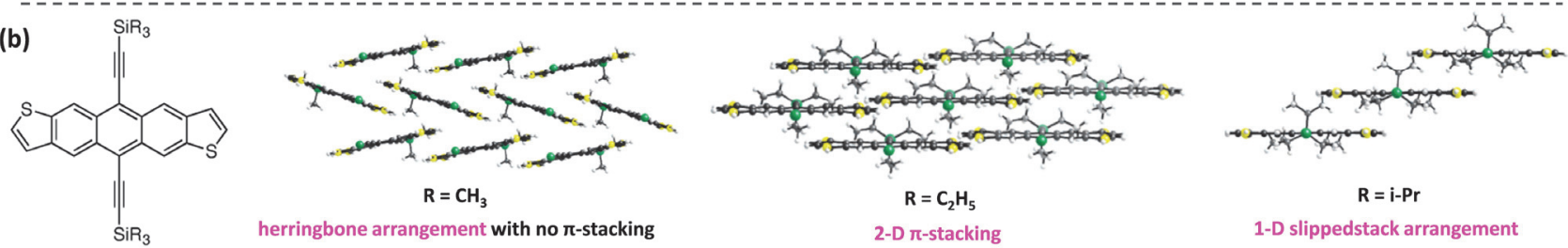

(c)
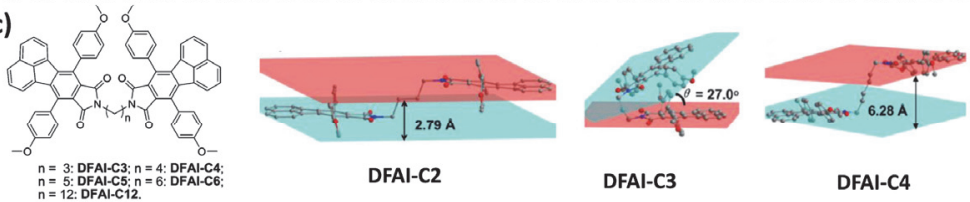

DFAI-C3

DFAI-C4

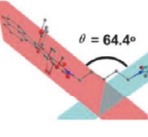

DFAI-C5

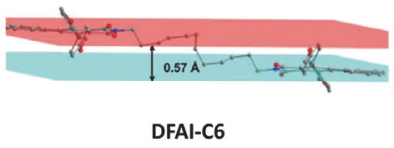

(d)
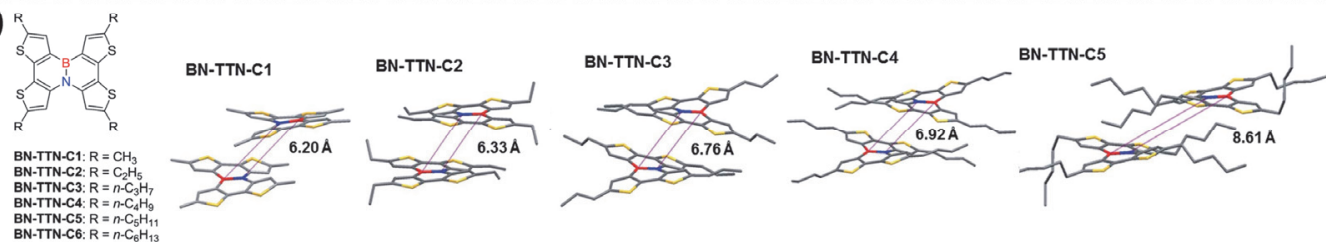

BN-TTN-C6

图 12 (a) 卤素对分子排列和 OFET 性能的影响 ${ }^{[99]}$; (b d) 烷基链长度对分子排列及 OFET 性能的影响 ${ }^{[103,105-106]}$

Figure 12 The influence of halogen ${ }^{[99]}$ (a) and alkyl chain length ${ }^{[103,105-106]}(\mathrm{b} \sim \mathrm{d})$ on molecular packing and OFET performance 
得到一系列 DFAI 衍生物(图 12c). 在晶体中, 偶数碳烷 基链的分子(DFAI-C4, DFAI-C6, DFAI-C12)呈现 “ $Z$ ” 型构象, 而奇数碳烷基链的分子(DFAI-C3, DFAI-C5)则 呈现 “ $\mathrm{V}$ ” 型构象, 其中, “ $\mathrm{Z}$ ” 型构象分子构成的排列, 分子间具有较弱的 $\pi \cdots \pi$ 相互作用, 从而导致离散的和非 定向的分子相互作用. 而 “V” 型构象分子构成的排列 会产生连续且定向的 $\pi \cdots \pi$ 重叠, 具有更强的一维生长趋 势. 随后, 他们在多环化合物 BN-TTN 上引入不同长度 的烷基链 ${ }^{[106]}$, 得到 6 个衍生物(BN-TTN-C1-6) (图 12d), 所有分子都表现出层状排列模式, 随着烷基链由甲基增 长为己基时, 相邻分子 $\mathrm{BN}$ 间距离由 $6.20 \AA$ 逐渐变大为 $9.21 \AA$, 并且, 随着烷基链增长, BN-TTN-C1 到 BN-TTN-C4 表现出了约 $3.44 \AA$ 的 $\pi \cdots \pi$ 相互作用, BN-TTN-C5 和 BN-TTN-C6 的 $\pi \cdots \pi$ 重叠几乎可以忽略. 结合分子的结晶性以及薄膜形貌, 6 个分子在器件性能 中表现出奇偶效应, 即奇数碳烷基链的分子表现出较高 的迁移率.

小分子中的电荷传输对分子间的 $\pi$ 轨道重叠非常敏 感, 分子堆积的任何细微变化都会改变 $\pi$ 轨道重叠, 从 而导致载流子传输的变化. 有机半导体的同质多晶型表 现出不同的分子排列和不同的迁移率, 进一步说明分子 排列对有机场效应晶体管的性能有重要影响 ${ }^{[107]}$. TTF 的同质多晶现象早在 1994 年就被发现 ${ }^{[108]}$, 直到 2007 年 $\mathrm{Hu}$ 等[109]才通过严格控制实验条件得到了单独的两种不 同晶型, 并详细研究了 TTF 的两种晶型对迁移率的影 响, 其中 $\alpha$-相晶体中具有较强的 $\pi \cdots \pi$ 相互作用, 体现出 相对较高的迁移率. 最近, $\mathrm{Hu}$ 等 ${ }^{[110]}$ 还研究报道了分子 DABD 的两种不同晶型(图 13a), 其中, $\alpha$-相晶体中分子 以二聚体的形式成对出现, 形成了典型的鱼骨状堆积模 式, 二聚体中 $\pi \cdots \pi$ 堆积距离为 $3.356 \AA . \beta$-相晶体中, 分 子也呈现鱼骨状堆积模式, $\pi \cdots \pi$ 重叠面积更大, 更有利 于载流子传输. 2015 年, Perepichka 等[111]报道了一种新 化合物 TT-TzTz-TT-1 并得到了红色针状晶体和黄色片 状晶体(图 $13 \mathrm{~b}$ ). 在晶体排列中, 红色针状晶体以 slip-stack 形式晶型排列, 晶面间距约为 $3.45 \AA$, 所形成
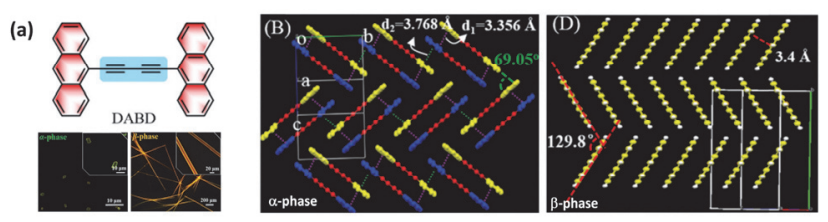

(b)
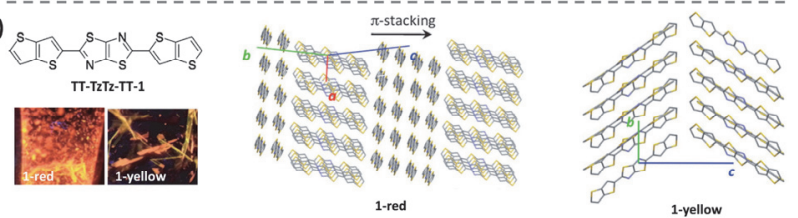

图 13 (a) 分子 $\mathbf{D A B D}{ }^{[110]}$ 和(b) TT-TzTz-TT-1 ${ }^{[111}$ 的同质多晶形、分子 排列及其 OFET 性能

Figure 13 Polymorphs, molecular packing and OFET properties of compound (a) DABD ${ }^{[110]}$ and (b) TT-TzTz-TT-1 ${ }^{[111]}$
的四聚体间呈现不连续的 $\pi \cdots \pi$ 堆积, 无法进行载流子传 输. 鱼骨状排列的黄色片状晶体则形成长程有序的 $\pi \cdots \pi$ 堆积, 晶面间距约为 $3.41 \AA$, 这种长程的 $\pi \cdots \pi$ 堆积更有 利于器件中载流子的传输, 因此晶体 1-yellow 具备载流 子传输效果.

\section{6 分子排列对其他性能的影响}

分子排列和取向及形貌对材料的光电转换效率和 性能也有重要影响 ${ }^{[112-115]}$. 例如, 在钲钛矿太阳能电池 中, 王鹏等 ${ }^{[116]}$ 通过取代基的微小改变, 调节了分子排 列. 其中, $\mathbf{A Z 1}$ 和 $\mathbf{A Z 2}$ 都呈现出反平行排列，与化合物 $\mathbf{A Z 1}$ 相比, 化合物 $\mathbf{A Z 2}$ 在沿分子短轴方向的滑移大大 减弱, 二聚体与二聚体间的距离更短(3.63 A), AZ2 的分 子排列特性是其具备较高的薄膜空穴迁移率的主要原 因, 从微观的分子排列上进一步为钙钛矿太阳能电池中 有机半导体的空穴传输机理提供了新的认识. Bäuerle 等 ${ }^{[117]}$ 通过改变共轭骨架上的烷基链得到了具备不同 $\pi \cdots \pi$ 堆积的有机小分子, 其中, DCV4T 2 具有较强的分 子间相互作用力和 $\pi \cdots \pi$ 相互作用, 形成了完美的平面层 状排列，这种排列同时也通过 GIXRD 得到证实. DCV4T-Me 2 的优异固态性能体现在电荷载流子迁移 率中, 与 DCV4T 1 和 DCV4T 3 相比, DCV4T-Me 2 表 现出最高的载流子迁移率, 达 $2 \times 10^{-4} \mathrm{~cm}^{2} \cdot \mathrm{V}^{-1} \cdot \mathrm{s}^{-1}$, 同 时, 也证明了有机小分子排列对有机太阳能电池效率的 影响.

\section{7 总结与展望}

本综述主要针对微观分子排列与宏观性质, 总结了 由单个分子通过分子间作用力构成的不同分子排列对 力致变色、室温磷光、力致发光、有机场效应晶体管以 及其他光电性能(如太阳能电池)的影响, 详细讨论了分 子结构的细微调节(电子效应、空间位阻效应、烷基链、 卤素等)对分子堆积的影响, 分析了分子排列与分子间 相互作用对不同光电性能的决定性作用, 进一步明确了 分子排列的重要性，为指导有机功能分子的设计提供有 用信息. 因此，在设计功能材料时，聚集态分子的排列 对性能的影响不容忽视. 基于此, $\mathrm{Li}$ 等 ${ }^{[88]}$ 在 2018 年提出 MUSIC (Molecular Uniting Set Identified Characteristic) 概念, 以音乐形象化分子的聚集态行为, 指出分子在聚 集态体现出的特性. 而调节聚集态分子排列, 是进一步 优化有机、高分子功能材料性能的又一重要策略 ${ }^{[106-117]}$.

众多研究已经强调了分子排列对性能的影响, 并初 步发现了一些规律: 以相对成熟的力致变色为例, 在外 力作用下, 分子由晶态转为无定型态或者分子堆积更为 紧密或是发生相变, 从而实现发光颜色变化. 而在力致 变色领域, 同时具有扭曲单元(AIE 单元)的 D-A 结构将 是理想的模型分子, D-A 结构影响分子的电子结构, 扭 曲单元则使分子在晶态中排列相对疏松, 在外力的作用 
下，分子排列易改变(或无定型化). 而烷基链、卤原子、 氧基以及含氧、氮等元素的基团则是构建聚集状态分子 间相互作用的有效构筑单元, 这些都仅仅是建立的初 步、大概的设计理念和模型. 从分子设计角度出发, 力 致发光分子的设计理念和力致变色类似, 但从仅有的机 理出发, 力致发光分子大多是非中心对称的具有压电效 应的分子. 而在室温磷光、有机场效应晶体管以及其他 光电材料中, 更关注的是分子间的 $\pi-\pi$ 堆积, 一方面 $\pi-\pi$ 堆积有助于分子形成紧密的 $H$ 型排列, 稳定三线态激 子; 另一方面, 有助于载流子的传输.

实际上, 分子结构决定分子的电子特性, 而在不同 刺激条件下单个分子的电子性质如何影响分子的排列 和构象知之甚少, 因此, 建立精确的结构-排列-性能关 系是充满机遇和挑战的.

由于目前测试技术和手段的缺乏, 无法监测到刺激 作用那一瞬间激发态分子构象和排列的变化, 故大部分 相关工作都是基于刺激作用前的分析和研究. 而另外一 方面, 对于刺激(压力等)的量化和刺激时相应的构象、 排列的变化, 缺乏系统的、实质性的研究, 无法为内在 机理的研究提供更多详细的信息, 这很大程度上限制了 精确的结构-堆积-性能关系的建立. 因此, 我们需要进 行更多的尝试, 尤其是那些精心设计并带有特定思想的 探究, 有助于从内源建立精确的结构-堆积-性能关系.

\section{作者简介}

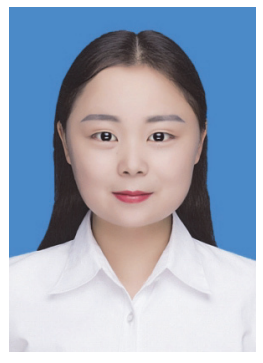

王金风, 湖北黄冈人, 博士研究生, 天津大学工程师, 2019 年于武汉大学获得理学博士学位, 主要从事染料敏化太 阳能电池、室温磷光和力致发光相关研究.

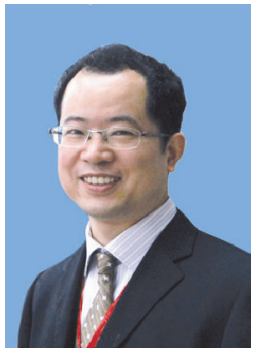

李振，湖北孝感人，教授，国家杰出青年科学基金获得者， “科技部中青年科技创新领军人才计划” 入选者, 国家 “万人 计划” 入选者, 英国皇家化学会会士(Fellow of RSC). 1997 年 于武汉大学化学系获理学学士学位, 2002 年于武汉大学化学 学院获理学博士学位. 研究方向为有机、高分子光电功能材料
化学. 研究对象主要为有机共轭体系和功能高分子, 研究范 围涉及二阶非线性光学、有机室温磷光、力致发光、聚集诱 导发光、传感器、太阳能电池、磁性纳米材料等.

\section{References}

[1] Mei, J.; Leung, N. L. C.; Kwok, R. T. K.; Lam, J. W. Y.; Tang, B. Z. Chem. Rev. 2015, 115, 11718.

[2] Zhi, J.; Zhou, Q.; Shi, H.; An, Z.; Huang, W. Chem. Asian J. 2020, $15,947$.

[3] Shi, H.; Zou, L.; Huang, K.; Wang, H.; Sun, C.; Wang, S.; Ma, H.; He, Y.; Wang, J.; Yu, H.; Yao, W.; An, Z.; Zhao, Q.; Huang, W. ACS Appl. Mater. Interfaces 2019, 11, 18103.

[4] Song, Y.; Xu, L.; Wu, Q.; Xiao, S.; Zeng, H.; Gong, Y.; Li, C.; Cheng, S.; Li, Q.; Zhang, L.; Li, Z. Small Methods 2020, 1900779.

[5] Zhang, H.; Huang, L.; Li, T.; Liu, B.; Bai, Z.; Li, X.; Lu, D. Acta Chim. Sinica 2019, 77, 397 (in Chinese). (张昊，黄龙，李涛，刘宾， 白泽明, 李小娜, 陆丹, 化学学报, 2019, 77, 397.)

[6] Jiang, Q.; Wang, Z.; Li, M.; Yang, Y.; Xu, X.; Xu, H.; Wang, S. Chin. J. Org. Chem. 2020, 40, 4290 (in Chinese). (姜倩, 王忠龙, 李明新, 杨益琴, 徐徐, 徐海军, 王石发, 有机化学, 2020, 40, 4290.)

[7] Yuan, W. Z.; Gong, Y.; Chen, S.; Shen, X. Y.; Lam, J. W. Y.; Lu, P.; Lu, Y.; Wang, Z.; Hu, R.; Xie, N.; Kwok, H. S.; Zhang, Y.; Sun, J. Z.; Tang, B. Z. Chem. Mater. 2012, 24, 1518.

[8] Yang, J.; Huang, J.; Li, Q.; Li, Z. J. Mater. Chem. C 2016, 4, 2663.

[9] Han, M.; Chen, Y.; Xie, Y.; Zhang, F.; Li, X.; Huang, A.; Fan, Y.; Fan, Y.; Gong, Y.; Peng, Q.; Li, Q.; Ma, D.; Li, Z. Cell Rep. Phys. Sci. 2020, 1, 100252.

[10] Miao, Q.; Xie, C.; Zhen, X.; Lyu, Y.; Duan, H.; Liu, X.; Jokerst, J. V.; Pu, K. Nat. Biotechnol. 2017, 35, 1102.

[11] He, Z.; Gao, H.; Zhang, S.; Zheng, S.; Wang, Y.; Zhao, Z.; Ding, D.; Yang, B.; Zhang, Y.; Yuan, W. Z. Adv. Mater. 2019, 31, 1807222.

[12] Li, Q.; Li, Z. Acc. Chem. Res. 2020, 53, 962.

[13] Li, Q.; Li, Z. Sci. China. Mater. 2020, 63, 177.

[14] Li, Q.; Li, Z. Adv. Sci. 2017, 4, 1600484.

[15] Yang, J.; Fang, M.; Li, Z. Aggregate 2020, 1, 6.

[16] Yang, J.; Chi, Z.; Zhu, W.; Tang, B. Z.; Li, Z. Sci. China Chem. 2019, 62, 1090.

[17] Liu, F.; Liao, Q.; Wang, J.; Gong, Y.; Dang, Q.; Ling, W.; Han, M.; Li, Q.; Li, Z. Sci. China Chem. 2020, 63, 1435.

[18] Dong, Y. Q.; Lam, J. W. Y.; Tang, B. Z. J. Phys. Chem. Lett. 2015, 6, 3429.

[19] Wang, C.; Li, Z. Mater. Chem. Front. 2017, 1, 2174.

[20] Yao, X.; Chi, Z. Sci. China. Chem. 2013, 43, 1090.

[21] Yang, Z.; Chi, Z.; Mao, Z.; Zhang, Y.; Liu, S.; Zhao, J.; Aldred, M. P.; Chi, Z. Mater. Chem. Front. 2018, 2, 861

[22] Ma, Z.; Wang, Z.; Meng, X.; Ma, Z.; Xu, Z.; Ma, Y.; Jia, X. Angew. Chem. Int. Ed. 2016, 55, 519.

[23] Yuan, W.; Tan, Y.; Gong, Y.; Lu, P.; Lam, J. W. Y.; Shen, X.; Feng, C.; Sung, H. H.-Y.; Lu, Y.; Williams, I. D.; Sun, J.; Zhang, Y.; Tang, B. Z. Adv. Mater. 2013, 25, 2837.

[24] Sagara, Y.; Yamane, S.; Mitani, M.; Weder, C.; Kato, T. Adv. Mater. 2016, 28, 1073 .

[25] Shen, X.; Wang, Y.; Zhao, E.; Yuan, W.; Liu, Y.; Lu, P.; Qin, A.; Ma, Y.; Sun, J.; Tang, B. Z. J. Phys. Chem. C 2013, 117, 7334.

[26] Zhang, X.; Chi, Z.; Zhang, J.; Li, H.; Xu, B.; Li, X.; Liu, S.; Zhang, Y.; Xu, J. J. Phys. Chem. B 2011, 115, 7606.

[27] Jadhav, T.; Dhokale, B.; Mobin, S. M.; Misra, R. J. Mater. Chem. C 2015, 3, 9981.

[28] Yang, J.; Li, L.; Yu, Y.; Ren, Z.; Peng, Q.; Ye, S.; Li, Q.; Li, Z. Mater. Chem. Front. 2017, 1, 91.

[29] Gong, Y. B.; Zhang, P.; Gu, Y. R.; Wang, J. Q.; Han, M. M.; Chen, C.; Zhan, X. J.; Xie, Z. L.; Zou, B.; Peng, Q.; Chi, Z. G.; Li, Z. Adv. Opt. Mater. 2018, 6, 1800198.

[30] Gong, Y.; He, S.; Li, Y.; Li, Z.; Liao, Q.; Gu, Y.; Wang, J.; Zou, B.; Li, Q.; Li, Z. Adv. Opt. Mater. 2020, 8, 1902036.

[31] Xue, S.; Qiu, X.; Sun, Q.; Yang, W. J. Mater. Chem. C 2016, 4, 1568.

[32] Wang, Y.; Liu, W.; Bu, L.; Li, J.; Zheng, M.; Zhang, D.; Sun, M.; Tao, Y.; Xue, S.; Yang, W. J. Mater. Chem. C 2013, $1,856$.

[33] Jiang, H.; Liu, X. J.; Jia, R. R.; Xu, T. H.; Xia, M. RSC. Adv. 2019, 9, 30381 .

[34] Zhang, X.; Chi, Z.; Xu, B.; Jiang, L.; Zhou, X.; Zhang, Y.; Liu, S.; 
Xu, J. Chem. Commun. 2012, 48, 10895.

[35] Bu, L.; Sun, M.; Zhang, D.; Liu, W.; Wang, Y.; Zheng, M.; Xue, S.; Yang, W. J. Mater. Chem. C 2013, 1, 2028.

[36] Yang, W.; Liu, C.; Lu, S.; Du, J.; Gao, Q.; Zhang, R.; Liu, Y.; Yang, C. J. Mater. Chem. C 2018, 6, 290.

[37] Lei, Y.; Zhou, Y.; Qian, L.; Wang, Y.; Liu, M.; Huang, X.; Wu, G.; Wu, H.; Ding, J.; Cheng, Y. J. Mater. Chem. C 2017, 5, 5183.

[38] He, Z.; Zhang, L.; Mei, J.; Zhang, T.; Lam, J. W. Y.; Shuai, Z.; Dong, Y. Q.; Tang, B. Z. Chem. Mater. 2015, 27, 6601.

[39] Dong, Y.; Xu, B.; Zhang, J.; Tan, X.; Wang, L.; Chen, J.; Lv, H.; Wen, S.; Li, B.; Ye, L.; Zou, B.; Tian, W. Angew. Chem. Int. Ed. 2012, 51, 10782 .

[40] Huang, B.; Chen, W. C.; Li, Z.; Zhang, J.; Zhao, W.; Feng, Y.; Tang, B. Z.; Lee, C. S. Angew. Chem. Int. Ed. 2018, 57, 12473.

[41] Xiong, Y.; Huang, J.; Liu, Y.; Xiao, B.; Xu, B.; Zhao, Z.; Tang, B. Z. J. Mater. Chem. C 2020, 8, 2460.

[42] Jhaa, P.; Chandra, B. P. Luminescence 2014, 29, 977.

[43] Xie, Y.; Li, Z. Mater. Chem. Front. 2020, 4, 317.

[44] Chang, K.; Li, Q.; Li, Z. Chin. J. Org. Chem. 2020, 40, 3656 (in Chinese). (常凯, 李倩倩, 李振, 有机化学, 2020, 40, 3656.)

[45] Xu, B.; He, J.; Mu, Y.; Zhu, Q.; Wu, S.; Wang, Y.; Zhang, Y.; Jin, C.; Lo, C.; Chi, Z.; Lien, A.; Liu, S.; Xu, J. Chem. Sci. 2015, 6, 3236.

[46] Wang, C.; Xu, B.; Li, M.; Chi, Z.; Xie, Y.; Li, Q.; Li, Z. Mater. Horiz. 2016, 3, 220.

[47] Xie, Y.; Tu, J.; Zhang, T.; Wang, J.; Xie, Z.; Chi, Z.; Peng, Q.; Li, Z. Chem. Commun. 2017, 53, 11330.

[48] Tu, J.; Liu, F.; Wang, J.; Li, X.; Gong, Y.; Fan, Y.; Han, M.; Li, Q.; Li, Z. ChemPhotoChem 2019, 3, 133 .

[49] Liu, F.; Tu, J.; Wang, X.; Wang, J.; Gong, Y.; Han, M.; Dang, X.; Liao, Q.; Peng, Q.; Li, Q.; Li, Z. Chem. Commun. 2018, 54, 5598.

[50] Xu, B.; Li, W.; He, J.; Wu, S.; Zhu, Q.; Yang, Z.; Wu, Y. C.; Zhang, Y.; Jin, C.; Lu, P. Y.; Chi, Z.; Liu, S.; Xu, J.; Bryce, M. R. Chem. Sci. 2016, 7, 5307 .

[51] Huang, G.; Jiang, Y.; Wang, J.; Li, Z.; Li, B. S.; Tang, B. Z. J. Mater. Chem. C 2019, 7, 12709.

[52] Jiang, Y.; Wang, J.; Huang, G.; Li, Z.; Li, B. S.; Tang, B. Z. J. Mater. Chem. C 2019, 7, 11790.

[53] Wang, C.; Yu, Y.; Chai, Z.; He, F.; Wu, C.; Gong, Y.; Han, M.; Li, Q.; Li, Z. Mater. Chem. Front. 2019, 3, 32.

[54] Wang, C.; Yu, Y.; Yuan, Y.; Ren, C.; Liao, Q.; Wang, J.; Chai, Z.; Li, Q.; Li, Z. Matter 2019, 2, 181.

[55] Xu, S.; Liu, T.; Mu, Y.; Wang, Y.; Chi, Z.; Lo, C.; Liu, S.; Zhang, Y.; Lien, A.; Xu, J. Angew. Chem. Int. Ed. 2015, 54, 874.

[56] Yang, J.; Gao, X.; Xie, Z.; Gong, Y.; Fang, M.; Peng, Q.; Chi, Z.; Li, Z. Angew. Chem. Int. Ed. 2017, 56, 15299.

[57] Yang, J.; Qin, J.; Geng, P.; Wang, J.; Fang, M.; Li, Z. Angew. Chem. Int. Ed. 2018, 130, 14370.

[58] Chen, Y.; Xu, C.; Xu, B.; Mao, Z.; Li, J. A.; Yang, Z.; Peethani, N. R.; Liu, C.; Shi, G.; Gu, F. L.; Zhang, Y.; Chi, Z. Mater. Chem. Front. 2019, 3, 1800.

[59] Neena, K. K.; Sudhakar, P.; Dipak, K.; Thilagar, P. Chem. Commun. 2017, 53, 3641 .

[60] Arivazhagan, C.; Maity, A.; Bakthavachalam, K.; Jana, A.; Panigrahi, S. K.; Suresh, E.; Das, A.; Ghosh, S. Chem. Eur. J. 2017, 23, 7046.

[61] Fang, M.; Yang, J.; Liao, Q.; Gong, Y.; Xie, Z.; Chi, Z.; Peng, Q.; Li, Q.; Li, Z. J. Mater. Chem. C 2017, 5, 9879.

[62] Yu, Y.; Wang, C.; Wei, Y.; Fan, Y.; Yang, J.; Wang, J.; Han, M.; Li, Q.; Li, Z. Adv. Opt. Mater. 2019, 7, 1900505.

[63] Tu, J.; Fan, Y.; Wang, J.; Li, X.; Liu, F.; Han, M.; Wang, C.; Li, Q.; Li, Z. J. Mater. Chem. C 2019, 7, 12256.

[64] Wang, J.; Chai, Z.; Wang J.; Wang, C.; Han, M.; Liao, Q.; Huang, A.; Lin, P.; Li, C.; Li, Q.; Li, Z. Angew. Chem. Int. Ed. 2019, 58, 17297.

[65] Yu, Y.; Fan, Y.; Wang, C.; Wei, Y.; Liao, Q.; Li, Q.; Li, Z. J. Mater. Chem. C 2019, 7, 13759.

[66] Liu, X.; Jia, Y.; Jiang, H.; Gao, G.; Xia, M. Acta Chim. Sinica 2019, 77, 1194 (in Chinese). (刘笑静, 贾彦荣, 江豪, 高贯雷, 夏敏, 化 学学报, 2019, 77, 1194.)

[67] Li, W.; Huang, Q.; Mao, Z.; Li. Q.; Jiang, L.; Xie, Z.; Xu, R.; Yang, Z.; Zhao, J.; Yu, T.; Zhang, Y.; Aldred, M. P.; Chi, Z. Angew. Chem. Int. Ed. 2018, 130, 12909.

[68] Sun, Q.; Zhang, K.; Zhang, Z.; Tang, L.; Xie, Z.; Chi, Z.; Xue, S.; Zhang, H.; Yang, W. Chem. Commun. 2018, 54, 8206.

[69] Yang, J.; Ren, Z.; Xie, Z.; Liu, Y.; Wang, C.; Xie, Y.; Peng, Q.; Xu,
B.; Tian, W.; Zhang, F.; Chi, Z.; Li, Q.; Li, Z. Angew. Chem. Int. Ed. 2017, 56, 880 .

[70] Wang, J.; Wang, C.; Gong, Y.; Liao, Q.; Han, M.; Jiang, T.; Dang, Q.; Li, Y.; Li, Q.; Li, Z. Angew. Chem. Int. Ed. 2018, 130, 17063.

[71] Li, J. A.; Zhou, J.; Mao, Z.; Xie, Z.; Yang, Z.; Xu, B.; Liu, C.; Chen, X.; Ren, D.; Pan, H.; Shi, G.; Zhang, Y.; Chi, Z. Angew. Chem. Int. Ed. 2018, 57, 6449 .

[72] Mukherjee, S.; Thilagar, P. Chem. Commun. 2015, 51, 10988.

[73] Forni, A.; Lucenti, E.; Botta, C.; Cariati, E. J. Mater. Chem. C 2018, $6,4603$.

[74] Ma, H.; Lv, A.; Fu, L.; Wang, S.; An, Z.; Shi, H.; Huang, W. Ann Phys. 2019, 531, 1800482.

[75] Wang, Y.; Yang, J.; Gong, Y.; Fang, M.; Li, Z.; Tang, B. Z. SmartMat 2020, 1, e1006.

[76] Yang, J.; Fang, M.; Li, Z. InfoMat 2020, 2, 791.

[77] Fang, M.; Yang, J.; Li, Z. Chinese J. Polym. Sci. 2019, 37, 383.

[78] Dang, Q.; Jiang, Y.; Wang, J.; Wang, J.; Zhang, Q.; Zhang, M.; Luo, S.; Xie, Y.; Pu, K.; Li, Q.; Li, Z. Adv. Mater. 2020, 32, 2006752.

[79] Wang,Y.; Yang, J.; Fang, M.; Yu, Y.; Zou, B.; Wang, L.; Tian, Y.; Cheng, J.; Tang, B.; Li, Z. Matter 2020, 2, 449.

[80] Yuan, W. Z.; Shen, X. Y.; Zhao, H.; Lam, J. W. Y.; Tang, L.; Lu, P.; Wang, C.; Liu, Y.; Wang, Z.; Zheng, Q.; Sun, J. Z.; Ma, Y.; Tang, B. Z. J. Phys. Chem. C 2010, 114, 6090.

[81] Zhang, L.; Li, M.; Gao, Q.; Chen, C. Chin. J. Org. Chem. 2020, 40, 516.

[82] An, Z.; Zheng, C.; Tao, Y.; Chen, R.; Shi, H.; Chen, T.; Wang, Z.; Li, H.; Deng, R.; Liu, X.; Huang, W. Nat. Mater. 2015, 14, 685.

[83] Chai, Z.; Wang, C.; Wang, J.; Liu, F.; Xie, Y.; Zhang, Y. Z.; Li, J. R.; Li, Q.; Li, Z. Chem. Sci. 2017, 8, 8336.

[84] Che, W.; Gong, Y.; Tu, L.; Han, M.; Li, X.; Xie, Y.; Li, Z. Phys Chem. Chem. Phys. 2020, 9, 1262.

[85] Yang, J.; Ren, Z.; Chen, B.; Fang, M.; Zhao, Z.; Tang, B.; Peng, Q.; Li, Z. J. Mater. Chem. C 2017, 5, 9242.

[86] Tian, Y.; Gong, Y.; Liao, Q.; Wang, Y.; Ren, J.; Fang, M.; Yang, J.; Li, Z. Cell Rep. Phys. Sci. 2020, 1, 100052.

[87] Yang, J.; Gao, H.; Wang,Y.; Yu, Y.; Gong, Y.; Fang, M.; Ding, D.; Hu, W.; Tang, B. Z.; Li, Z. Mater. Chem. Front. 2019, 3, 1391.

[88] Yang, J.; Zhen, X.; Wang, B.; Gao, X.; Ren, Z.; Wang, J.; Xie, Y.; Li, J.; Peng, Q.; Pu, K.; Li, Z. Nat. Commun. 2018, 9, 840.

[89] Dang, Q.; Hu, L.; Wang, J.; Zhang, Q.; Han, M.; Luo, S.; Gong, Y.; Wang, C.; Li, Q.; Li, Z. Chem. Eur. J. 2019, 25, 7031.

[90] Liao, Q.; Gao, Q.; Wang, J.; Gong, Y.; Peng, Q.; Tian, Y.; Fan, Y.; Guo, H.; Ding, D.; Li, Q.; Li, Z. Angew. Chem. Int. Ed. 2020, 59, 9946.

[91] Fang, M.; Yang, J.; Xiang, X.; Xie, Y.; Dong, Y.; Peng, Q.; Li, Q.; Li, Z. Mater. Chem. Front. 2018, 2, 2124.

[92] Zheng, S.; Zhu, T.; Wang, Y.; Yang, T.; Yuan, W. Z. Angew. Chem. Int. Ed. 2020, 59, 10018.

[93] Wang, C.; Dong, H.; Hu, W.; Liu, Y.; Zhu, D. Chem. Rev. 2012, 112, 2208 .

[94] Yu, P.; Zhen, Y.; Dong, H.; Hu, W. Chem 2019, 5, 2814.

[95] Wang, C.; Dong, H.; Jiang, L.; Hu, W. Chem. Soc. Rev. 2018, 47, 422.

[96] Dong, H.; Fu, X.; Liu, J.; Wang, Z.; Hu, W. Adv. Mater. 2013, 25, 6158 .

[97] $\mathrm{Hu}, \mathrm{W}$. Organic field effect transistor, Science press, Beijing, 2011 (in Chinese). (胡文平, 有机场效应晶体管, 科学出版社, 北京, 2011.)

[98] Moon, H.; Zeis, R.; Borkent, E.; Besnard, C.; Lovinger, A.; Siegrist, T.; Kloc, C.; Bao, Z. J. Am. Chem. Soc. 2004, 126, 15322.

[99] Dou, J.; Zheng, Y.; Yao, Z.; Yu, Z.; Lei, T.; Shen, X.; Luo, X.; Sun, J.; Zhang, S.; Ding, Y.; Han, G.; Yi, Y.; Wang, J.; Pei, J. J. Am. Chem. Soc. 2015, 137, 15947

[100] Lei, T.; Wang, J.; Pei, J. Chem. Mater. 2014, 26, 594.

[101] Wang, C.; Qin, Y.; Sun, Y.; Guan, Y.; Xu, W.; Zhu, D. ACS Appl. Mater. Interfaces 2015, 7, 15978.

[102] Yassar, A. Polym. Sci. Ser. C 2014, 56, 4.

[103] Payne, M.; Parkin, S.; Anthony, J.; Kuo, C.; Jackson, T. J. Am. Chem. Soc. 2005, 127, 4986.

[104] Zhan, X.; Zhang, J.; Gong, Y.; Tang, S.; Tu, J.; Xie, Y.; Peng, Q.; Yu, G.; Li, Z. Mater. Chem. Front. 2017, 1, 2341.

[105] Ding, L.; Li, H.; Lei, T.; Ying, H.; Wang, R.; Zhou, Y.; Su, Z.; Pei, J. Chem. Mater. 2012, 24, 1944.

[106] Wang, X.; Zhuang, F.; Zhou, X.; Yang, D.; Wang, J.; Pei, J. J. Mater Chem. C 2014, 2, 8152 
[107] Chung, H.; Diao, Y. J. Mater. Chem. C 2016, 4, 3915.

[108] Ellern, A.; Bernstein, J.; Becker, J.; Zamir, S.; Shahal, L. Chem Mater. 1994, 6, 1378.

[109] Jiang, H.; Yang, X.; Cui, Z.; Liu, Y.; Li, H.; Hu, W.; Liu, Y.; Zhu, D. Appl. Phys. Lett. 2007, 91, 123505.

[110] Liu, D.; Li, C.; Niu, S.; Li, Y.; Hu, M.; Li, Q.; Zhu, W.; Zhang, X.; Dong, H.; Hu, W. J. Mater. Chem. C 2019, 7, 5925.

[111] Schneider, J.; Black, H.; Lin, H.; Perepichka, D. ChemPhysChem 2015, 16, 1173.

[112] Li, Y.; Han, M.; Yang, W.; Guo, J.; Chang, K.; Wang, J.; Min, J.; Li, Q.; Li, Z. Mater. Chem. Front. 2019, 3, 1840.

[113] Gong, Y.; Chang, K.; Chen, C.; Han, M.; Zhan, X.; Min, J.; Jiao, X.;
Li, Q.; Li, Z. Mater. Chem. Front. 2019, 3, 93

[114] Liu, F.; Bi, S.; Wang, X.; Leng, X.; Han, M.; Xue, B.; Li, Q.; Zhou, H.; Li, Z. Sci. China Chem. 2019, 62, 739.

[115] Jin, M.; Zhu, Z.; Liao, Q.; Li, Q.; Li, Z. Chinese J. Polym. Sci. 2020 $38,118$.

[116] Zheng, A.; Wang, J.; Xu, N.; Zhu, R.; Yuan, Y.; Zhang, J.; Zhang, J.; $\mathrm{Li}, \mathrm{Z}$.; Wang, P. ACS Photonics 2018, 5, 4694.

[117] Fitzner, R.; Elschner, C.; Weil, M.; Uhrich, C.; Körner, C.; Riede, M.; Leo, K.; Pfeiffer, M.; Reinold, E.; Osteritz, E.; Bäuerle, P. $A d v$ Mater. 2012, 24, 675.

(Cheng, B.) 